\title{
A sectional characterization of the Dade group
}

\author{
Serge Bouc and Jacques Thévenaz
}

\begin{abstract}
Let $k$ be a field of characteristic $p$, let $P$ be a finite $p$ - group, where $p$ is an odd prime, and let $D(P)$ be the Dade group of endo-permutation $k P$-modules. It is known that $D(P)$ is detected via deflation-restriction by the family of all sections of $P$ which are elementary abelian of rank $\leq 2$. In this paper, we improve this result by characterizing $D(P)$ as the limit (with respect to deflationrestriction maps and conjugation maps) of all groups $D(T / S)$ where $T / S$ runs through all sections of $P$ which are either elementary abelian of rank $\leq 3$ or extraspecial of order $p^{3}$ and exponent $p$.
\end{abstract}

AMS Subject classification : 20C20

Keywords : endo-permutation module, Dade group, limit.

\section{Introduction}

Endo-permutation modules for finite $p$-groups play an important role in the representation theory of finite groups and were classified recently in Bo4. The set of equivalence classes of such modules is an abelian group $D(P)$ (with respect to tensor product), called the Dade group of $P$. An important ingredient for the classification is a detection theorem, proved in [CaTh1, which asserts (for odd $p$ ) that the product of all deflation-restriction maps

$$
\prod_{(T, S)} \operatorname{Defres}_{T / S}^{P}: D(P) \longrightarrow \prod_{(T, S)} D(T / S)
$$

is injective, where $T / S$ runs through all sections of $P$ which are elementary abelian of rank $\leq 2$. The purpose of this paper is to improve this result and characterize the image of the injective map above, but actually by changing slightly the target.

In order to motivate our result, let us first mention two classical cases where a similar situation occurs. The first instance is group cohomology $H^{*}(G, k)$ where $G$ is a finite group anf $k$ is a field of characteristic $p$. An easy detection result asserts that the restriction map $H^{*}(G, k) \rightarrow H^{*}(P, k)$ is injective where $P$ is a Sylow $p$-subgroup of $G$. This is then improved by characterizing the image of $H^{*}(G, k)$ as the set of $G$-stable elements in $H^{*}(P, k)$. This improvement can be stated in a more sophisticated way : $H^{*}(G, k)$ is isomorphic to the limit ${\underset{L}{Q}}_{\lim } H^{*}(Q, k)$ where $Q$ runs through all $p$-subgroups of $G$ and the limit is taken with respect to restrictions and conjugations.

The second classical case is the ordinary representation $\operatorname{ring} R(G)$ of a finite group $G$. This is detected on restriction to cyclic subgroups, but in order to characterize the image, Brauer had to introduce the larger class of Brauer-elementary subgroups. Brauer's theorem can be stated as follows : $R(G)$ is isomorphic to the limit $\underset{Q}{\underset{\lim }{(}} R(Q)$ where $Q$ runs through all Brauer-elementary subgroups of $G$ and the limit is taken with respect to restrictions and conjugations.

We are facing a similar situation with the Dade group, except that the detection map is not a product of restriction maps to subgroups, but a product of deflationrestriction maps to sections of the group. When $p$ is odd, the detection family consists of elementary abelian $p$-groups of rank $\leq 2$. However, as in the case of the ordinary representation ring, we need to enlarge this family to impose just the right conditions 
for the limit. It turns out that we have to include also elementary abelian $p$-groups of rank 3 and extraspecial groups of order $p^{3}$ and exponent $p$. Our main result is the following.

1.1. Theorem. Let $p$ be an odd prime number and $P$ a finite $p$-group. Then the natural map

$$
\prod_{(T, S)} \operatorname{Defres}_{T / S}^{P}: D(P) \longrightarrow \varliminf_{(T, S)} D(T / S)
$$

is an isomorphism, where $T / S$ runs through all sections of $P$ which are either elementary abelian $p$-groups of rank $\leq 3$ or isomorphic to the extraspecial group of order $p^{3}$ and exponent $p$.

As in earlier work (BoTh1, Bo4 $)$, the Dade group is viewed as a functor on $p$ groups with morphisms being compositions of restrictions, tensor inductions, deflations, inflations, and isomorphisms. In more technical terms, $D(-)$ is viewed as a biset functor and this plays a role again here.

More precisely, whenever $\mathcal{Y}$ is a class of $p$-groups closed under taking sections, one can consider biset functors defined only on $\mathcal{Y}$, with values in abelian groups. Let $\mathcal{F}_{\mathcal{Y}}$ be the category of all such functors (an abelian category) and write simply $\mathcal{F}$ for the category of functors defined on all finite $p$-groups. There is an obvious forgetful functor

$$
\mathcal{O}_{\mathcal{Y}}: \mathcal{F} \rightarrow \mathcal{F}_{\mathcal{Y}}
$$

and we consider its left and right adjoints

$$
\mathcal{L}_{\mathcal{Y}}: \mathcal{F}_{\mathcal{Y}} \rightarrow \mathcal{F} \text { and } \quad \mathcal{R}_{\mathcal{Y}}: \mathcal{F}_{\mathcal{Y}} \rightarrow \mathcal{F}
$$

The connection with limits is the following.

1.2. Theorem. With the notation above, for any functor $M \in \mathcal{F}_{\mathcal{Y}}$, we have :

$$
\mathcal{L}_{\mathcal{Y}} M(P) \cong \varliminf_{(T, S) \in \mathcal{Y}(P)} M(T / S) \quad \text { and } \quad \mathcal{R}_{\mathcal{Y}} M(P) \cong \varliminf_{(T, S) \in \mathcal{Y}(P)} M(T / S)
$$

where $\mathcal{Y}(P)$ denotes the set of pairs $(T, S)$ of subgroups of $P$ such that $S \unlhd T$ and $T / S \in \mathcal{Y}$.

This type of result is more or less standard in category theory. In our case, it is made explicit in an appendix at the end of the paper. The main point is that the various inverse limits can be organized to yield a functor, namely $\mathcal{R}_{\mathcal{Y}} M$. Therefore we can use the machinery of functors throughout this paper.

Using this point of view, the natural map of Theorem 1.1 becomes the unit morphism of the adjunction and our main result takes the following form.

1.3. Theorem. Let $p$ be an odd prime number and let $D$ be the Dade functor. Let $\mathcal{X}_{3}$ be the class of all p-groups which are either elementary abelian p-groups of rank $\leq 3$ or isomorphic to the extraspecial group of order $p^{3}$ and exponent $p$. Then the unit morphism

is an isomorphism.

$$
\eta_{D}^{\mathcal{X}_{3}}: D \rightarrow \mathcal{R}_{\mathcal{X}_{3}} \mathcal{O}_{\mathcal{X}_{3}} D
$$

Let us now describe the main ingredients of the proof. We consider first the class $\mathcal{X}$ of all $p$-groups which are either elementary abelian $p$-groups (without condition on the 
rank) or extraspecial groups of order $p^{3}$ and exponent $p$. We prove the theorem for $\mathcal{X}$ instead of $\mathcal{X}_{3}$ and then we use a technical lemma about elementary abelian groups to derive the more precise result for $\mathcal{X}_{3}$.

Let $D_{\text {tors }}$ be the torsion subfunctor of $D$. It follows from Bo3] and Bo4 that $D / D_{\text {tors }}$ is isomorphic to the $\mathbb{Z}$-dual $K^{*}$ of the functor $K=\operatorname{Ker}\left(B \rightarrow R_{\mathbb{Q}}\right)$, where $B$ is the Burnside functor and $R_{\mathbb{Q}}$ is the rational representation functor. Thus we have an exact sequence

$$
0 \longrightarrow D_{\text {tors }} \longrightarrow D \longrightarrow K^{*} \longrightarrow 0
$$

and we consider seperately $D_{\text {tors }}$ and $K^{*}$.

For $D_{\text {tors }}$, a detection theorem from CaTh1 together with a result from BoTh2 about elementary abelian groups imply that the unit morphism

$$
\eta_{D_{\text {tors }}}^{\mathcal{X}}: D_{\text {tors }} \longrightarrow \mathcal{R}_{\mathcal{X}} \mathcal{O}_{\mathcal{X}} D_{\text {tors }}
$$

is an isomorphism. Turning now to $K^{*}$, we use an induction theorem for $K$ which is proved in Bo4 (and which plays a crucial role for the classification of endo-permutation modules). This theorem implies that the counit map $\mathcal{L}_{\mathcal{X}} \mathcal{O}_{\mathcal{X}} K \rightarrow K$ is surjective (and it is here that the extraspecial group of order $p^{3}$ is necessary). Dualizing and using again the result from [BoTh2], we obtain that the unit morphism

$$
\eta_{K^{*}}^{\mathcal{X}}: K^{*} \longrightarrow \mathcal{R}_{\mathcal{X}} \mathcal{O}_{\mathcal{X}} K^{*}
$$

is an isomorphism. The main theorem follows from the two isomorphisms.

Our methods do not work when $p=2$ for several reasons. First $D$ is not a biset functor if $p=2$. Moreover, the detection map to elementary abelian sections is not injective in general when $p=2$ and one needs to add the cyclic group of order 4 and the quaternion group of order 8 in the detecting family. These two problems are not essential and the arguments could probably be modified accordingly, but there is one more problem : The result from BoTh2 concerning elementary abelian groups is about $p$-torsion and is used for the 2-torsion group $D_{\text {tors }}$. This collapses when $p=2$.

Finally, let us mention that the main result of this article plays a crucial role in a forthcoming paper of the first author [Bo5].

\section{Biset functors}

Biset functors play a key role throuhout this paper. We collect in this section the material needed later concerning those functors. In particular, we revisit an induction theorem for one specific functor, called $K$, which already was important for the classification of endo-permutation modules in [Bo4] and which is used again in an essential way in the present paper.

If $Q$ and $P$ are $p$-groups, a $(Q, P)$-biset is a finite set $U$ with a left action of $Q$ and a right action of $P$, such that $(x \cdot u) \cdot y=x \cdot(u \cdot y)$ for all $x \in Q, y \in P, u \in U$. We let $\mathcal{C}_{p}$ be the category of all finite $p$-groups with morphisms defined by :

$$
\operatorname{Hom}_{\mathcal{C}_{p}}(P, Q)=B\left(Q \times P^{o p}\right)
$$

the Burnside group of all finite $(Q, P)$-bisets. The composition of morphisms is $\mathbb{Z}$-linear and is induced by the usual product of bisets, namely $V \circ U=V \times{ }_{Q} U$ whenever $V$ is 
an $(R, Q)$-biset and $U$ is a $(Q, P)$-biset. A biset functor is an additive functor from $\mathcal{C}_{p}$ to the category $\mathcal{A} b$ of abelian groups (see Bo1 and [BoTh1]).

We now recall how, for suitable choices of bisets $U$, we obtain morphisms of restriction, induction, inflation, deflation, and isomorphisms. The word 'section' was used informally in the introduction, but we now give a more precise definition. A section of a group $P$ is a pair $(T, S)$ of subgroups of $P$ such that $S \unlhd T$. The group $T / S$ will be referred to as a subquotient of $P$. If $F$ is a biset functor and $(T, S)$ is a section of $P$, then the following homomorphisms are defined:

- $\operatorname{Inf}_{T / S}^{T}: F(T / S) \rightarrow F(T)$, induced by the $(T, T / S)$-biset $T / S$ (inflation);

- $\operatorname{Ind}_{\mathcal{T}}^{P}: F(T) \rightarrow F(P)$, induced by the $(P, T)$-biset $P$ (induction);

- $\operatorname{Def}_{T / S}^{T}: F(T) \rightarrow F(T / S)$, induced by the $(T / S, T)$-biset $S \backslash T$ (deflation);

- $\operatorname{Res}_{T}^{P}: F(P) \rightarrow F(T)$, induced by the $(T, P)$-biset $P$ (restriction);

- Whenever $\alpha: P \rightarrow Q$ is an isomorphism, the corresponding isomorphism Iso $_{\alpha}$ : $F(P) \rightarrow F(Q)$ is induced by the $(Q, P)$-biset $P$ with left action of $Q$ via $\alpha^{-1}$. In particular, $\operatorname{Conj}_{x}: F(T / S) \rightarrow F\left({ }^{x} T /{ }^{x} S\right)$ (where $\left.x \in P\right)$ is induced by the $\left({ }^{x} T /{ }^{x} S, T / S\right)$ biset $T / S$ (conjugation by $x$ ).

It can be shown that any transitive biset can be decomposed as a product of the above five types of bisets, namely a composition of a restriction, a deflation, an isomorphism, an inflation, and an induction (see Lemma 7.4 in BoTh1 or Lemma 3 in [Bo1]), so that a biset functor is essentially a functor endowed with those five types of morphisms. We also consider the composites :

- $\operatorname{Indinf}_{T / S}^{P}=\operatorname{Ind}_{T}^{P} \operatorname{Inf}_{T / S}^{T}$, induced by the $(P, T / S)$-biset $P \times_{T}(T / S) \cong P / S$;

- $\operatorname{Defres}_{T / S}^{P}=\operatorname{Def}_{T / S}^{T} \operatorname{Res}_{T}^{P}$, induced by the $(T / S, P)$-biset $(S \backslash T) \times_{T} P \cong S \backslash P$.

If $F$ is a biset functor and $x \in F(Q)$ for some fixed $p$-group $Q$, the subfunctor $F_{x}$ generated by $x$ is the intersection of all subfunctors $E$ of $F$ such that $x \in E(Q)$. Equivalently

$$
F_{x}(P)=\operatorname{Hom}_{\mathcal{C}_{p}}(Q, P) \times_{Q} x,
$$

and this means in practice that we have to consider linear combinations of elements obtained by applying to $x$ successively restrictions, deflations, isomorphisms, inflations, and inductions in order to get all possible elements of $F_{x}(P)$.

Some standard constructions give rise to biset functors. This is the case in particular for the Burnside group $B(P)$ and the rational representation group $R_{\mathbb{Q}}(P)$, which turn out to have strong connections with the Dade group, as we shall see in the next section.

Recall that $B(P)$ is the Grothendieck group of finite $P$-sets, with $\mathbb{Z}$-basis consisting of all transitive $P$-sets $P / S$, where $S$ runs over all subgroups of $P$ up to conjugation. For every $(Q, P)$-biset $U$, we have a map $B(U): B(P) \rightarrow B(Q)$ defined by $X \mapsto U \times_{P} X$ for every $P$-set $X$. This provides a structure of biset functor on $B$, called simply the Burnside functor. Note that if the morphism $U$ is a restriction, induction, inflation, or deflation, then the morphism $B(U)$ is indeed the corresponding morphism between Burnside groups.

On the other hand $R_{\mathbb{Q}}(P)$ is the Grothendieck group of all $\mathbb{Q} P$-modules (that is, the rational representations of $P$ ) and the irreducible rational representations form a $\mathbb{Z}$-basis of $R_{\mathbb{Q}}(P)$. For every $(Q, P)$-biset $U$, we have a map $R_{\mathbb{Q}}(P) \rightarrow R_{\mathbb{Q}}(Q)$ defined by $V \mapsto \mathbb{Q} U \otimes_{\mathbb{Q} P} V$ for every $\mathbb{Q} P$-module $V$ (where $\mathbb{Q} U$ is the permutation $(\mathbb{Q} Q, \mathbb{Q} P$ )bimodule with $\mathbb{Q}$-basis $U$ ). Thus $R_{\mathbb{Q}}$ is a biset functor and again we recover the usual morphisms of restriction, induction, inflation, or deflation for representation groups.

There is a natural morphism $B \rightarrow R_{\mathbb{Q}}$, which is surjective for $p$-groups by a result 
of Ritter and Segal. We define

$$
K=\operatorname{Ker}\left(B \rightarrow R_{\mathbb{Q}}\right) .
$$

This is a subfunctor of $B$ which plays a crucial role in the sequel. We shall need in particular the following induction theorem for the biset functor $K$, proved in Section 6 of [Bo4]. We also include some additional results which are only implicit in [Bo4].

2.1. Theorem. Let $p$ be an odd prime, let $\mathcal{E}_{2}$ be the class of all elementary abelian p-groups of rank $\leq 2$, and let $\mathcal{X}_{2}$ be the class of p-groups consisting of $\mathcal{E}_{2}$ and the groups isomorphic to the extraspecial p-group $X$ of order $p^{3}$ and exponent $p$.

(a) $K$ is generated by some specific element $\delta \in K(X)$. In particular, for every p-group $P$, the sum of all $\operatorname{Indinf}_{T / S}^{P} K(T / S)$, where $(T, S) \in \mathcal{X}_{2}(P)$, is equal to $K(P)$.

(b) Let $E$ be an elementary abelian group of order $p^{2}$ and let $\epsilon$ be a generator of $K(E) \cong \mathbb{Z}$. For every p-group $P$, the sum of all $\operatorname{Indinf}_{T / S}^{P} K(T / S)$, where $(T, S) \in$ $\mathcal{E}_{2}(P)$, is equal to $K_{\epsilon}(P)$, where $K_{\epsilon}$ is the subfunctor of $K$ generated by $\epsilon$.

(c) We have $p \cdot K(P) \subseteq K_{\epsilon}(P)$, for every p-group $P$.

Proof. (a) This is exactly Theorem 6.12 and Corollary 6.16 in Bo4.

(b) In the Burnside group $B(E)$, define the element

$$
\epsilon=E / 1-\sum_{\substack{F \leq E \\|F|=p}} E / F+p E / E .
$$

It is easy to see that the corresponding rational representation is zero, so that $\epsilon \in K(E)$. More precisely, $K(E) \cong \mathbb{Z}$ generated by $\epsilon$, because $B(E)$ is $\mathbb{Z}$-free of rank $p+3$ and $R_{\mathbb{Q}}(E)$ is $\mathbb{Z}$-free of rank $p+2$ (which is the number of cyclic subgroups of $E$ ).

Define $K_{\epsilon}$ to be the subfunctor of $K$ generated by $\epsilon$. On evaluation at a $p$-group $P$, we have

$$
K_{\epsilon}(P)=\sum_{(T, S) \in \mathcal{E}_{2}(P)} \operatorname{Indinf}_{T / S}^{P} K(T / S)
$$

because $K(Q)=0$ for any group $Q$ of order 1 or $p$ so that any proper deflation or restriction of $\epsilon$ is zero.

(c) Since $K$ is generated by $\delta$, the inclusion $p K \subseteq K_{\epsilon}$ will follow if we prove that $p \delta$ belongs to $K_{\epsilon}(X)$. The expression for $\delta$ in the Burnside group $B(X)$ is the following (see 6.9 of $[\mathrm{Bo} 4])$ :

$$
\delta=X / I-X / I Z-X / J+X / J Z,
$$

where $I$ and $J$ are non-conjugate subgroups of order $p$ and $Z$ is the centre of $X$. Now $I Z$ is elementary abelian of order $p^{2}$ and the subgroup $I$ has $p$ conjugates in $X$ contained in $I Z$ (the only additional subgroup of order $p$ in $I Z$ being $Z$ ). Therefore, if $\epsilon_{I Z}$ denotes $\epsilon$ viewed in $B(I Z)$, we have

$$
\operatorname{Ind}_{I Z}^{X} \epsilon_{I Z}=X / 1-X / Z-p X / I+p X / I Z
$$

and similarly when $I$ is replaced by $J$. It follows that

$$
\operatorname{Ind}_{J Z}^{X} \epsilon_{J Z}-\operatorname{Ind}_{I Z}^{X} \epsilon_{I Z}=p \delta
$$


and this shows that $p \delta \in K_{\epsilon}(X)$.

We end this section by considering duality (defined in [Bo3]). The structure of biset functor on a dual uses opposite bisets as follows. If $F$ is a biset functor, define $F^{*}(P)=\operatorname{Hom}_{\mathbb{Z}}(F(P), \mathbb{Z})$ for every $p$-group $P$. If $U$ is a $(Q, P)$-biset, then define $F^{*}(U): F^{*}(P) \rightarrow F^{*}(Q)$ to be the transpose

$$
F^{*}(U)=F\left(U^{o p}\right)^{t},
$$

where $U^{o p}$ denotes the $(P, Q)$-biset with underlying set equal to $U$ and left $P$-action of $x \in P$ defined by using the given right action of $x^{-1}$ on $U$, and similarly for the action of $Q$. The effect of this definition is that restriction for $F^{*}$ is the transpose of induction for $F$ (and conversely), while inflation for $F^{*}$ is the transpose of deflation for $F$ (and conversely).

By definition of $K$ and by the result of Ritter and Segal, we have an exact sequence

$$
0 \longrightarrow K \longrightarrow B \longrightarrow R_{\mathbb{Q}} \longrightarrow 0,
$$

and since we get free $\mathbb{Z}$-modules on each evaluation, we obtain by duality an exact sequence

$$
0 \longrightarrow R_{\mathbb{Q}}^{*} \longrightarrow B^{*} \longrightarrow K^{*} \longrightarrow 0
$$

This exact sequence has an intimate connection with the Dade functor, as we shall recall in the next section.

\section{The Dade functor}

We collect in this section the various results we need about the Dade group and the Dade functor. Let $p$ be a prime number (which will soon be assumed to be odd) and let $k$ be a field of characteristic $p$. For any finite $p$-group $P$, we let $D(P)$ be the Dade group of $P$, that is, the group of equivalence classes of endo-permutation $k P$-modules (see [BoTh1] for details). If $X$ is a non empty finite $P$-set, the kernel of the augmentation map $k X \rightarrow k$ is called a relative syzygy of the trivial module (relative to $X$ ) and is an endo-permutation module. These are the main examples of endo-permutation modules and we let $D^{\Omega}(P)$ denote the subgroup of $D(P)$ generated by relative syzygies of the trivial module.

Recall that if $(T, S)$ is a section of $P$, we have a restriction map $\operatorname{Res}_{T}^{P}: D(P) \rightarrow$ $D(T)$ and a tensor induction map $\operatorname{Ten}_{T}^{P}: D(T) \rightarrow D(P)$, as well as an inflation map $\operatorname{Inf}_{T / S}^{T}: D(T / S) \rightarrow D(T)$ and a deflation map $\operatorname{Def}_{T / S}^{T}: D(T) \rightarrow D(T / S)$. We also write $\operatorname{Defres}_{T / S}^{P}=\operatorname{Def}_{T / S}^{T} \operatorname{Res}_{T}^{P}$ and similarly $\operatorname{Teninf}_{T / S}^{P}=\operatorname{Ten}_{T}^{P} \operatorname{Inf}_{T / S}^{T}$. It is proved in $\left[\mathrm{Bo} 2\right.$ that $D^{\Omega}$ is invariant under those operations.

The main detection theorem which we are concerned with is the following (Theorem 13.1 in [CaTh1]). 
3.1. Theorem. Let $\mathcal{E}_{2}(P)$ be the set of sections $(T, S)$ of $P$ such that $T / S$ is elementary abelian of rank $\leq 2$. If $p$ is odd, the map

$$
\prod_{(T, S) \in \mathcal{E}_{2}(P)} \operatorname{Defres}_{T / S}^{P}: D(P) \longrightarrow \prod_{(T, S) \in \mathcal{E}_{2}(P)} D(T / S)
$$

is injective.

When $p$ is odd, we want to view $D$ as a biset functor. Any $(Q, P)$-biset $U$ induces a group homomorphism $D(U): D(P) \rightarrow D(Q)$ (see Corollary 2.13 of [BoTh1]). For the specific choices of bisets $U$ described in Section 2, we recover the above morphisms of restriction, tensor induction, inflation, deflation, and isomorphisms (see Section 2 of [BoTh1]).

\subsection{Proposition.}

(a) $D^{\Omega}$ is a biset functor.

(b) If $p$ is odd, $D$ is a biset functor. Moreover, $D_{\text {tors }}$ is a subfunctor of $D$, where $D_{\text {tors }}(P)$ denotes the torsion subgroup of $D(P)$.

Proof. (a) The proof that $D^{\Omega}$ is a biset functor is the starting point of $\mathrm{Bo}$ ].

(b) There are two ways of proving that $D$ is a biset functor. If $p$ is odd, we have $D^{\Omega}(P)=D(P)$ by Theorem 7.7 of [Bo4, which is one of the main theorems of the classification of endo-permutation $k P$-modules. Thus $D=D^{\Omega}$ and $D$ is a biset functor.

The other proof does not require the full classification but uses Theorem 3.1 . This approach is explicit in Theorem 10.1 of Th].

The fact that $D_{\text {tors }}$ is a subfunctor is clear since a torsion element is mapped to a torsion element by a group homomorphism.

Now we recall the connection between $D$ and the standard biset functors considered in Section 2.

3.3. Theorem. Let $p$ be an odd prime.

(a) The natural injection $R_{\mathbb{Q}}^{*} \rightarrow B^{*}$ fits into an exact sequence of biset functors

$$
0 \longrightarrow R_{\mathbb{Q}}^{*} \longrightarrow B^{*} \longrightarrow D^{\Omega} / D_{\text {tors }}^{\Omega} \longrightarrow 0 \text {. }
$$

(b) There is an exact sequence of biset functors

$$
0 \longrightarrow D_{\text {tors }} \longrightarrow D \longrightarrow K^{*} \longrightarrow 0 \text {. }
$$

Proof. (a) This exactly Theorem 1.8 of Bo3.

(b) Since $p$ is odd, we have $D^{\Omega}=D$ by Theorem 7.7 of [Bo4]. Comparing the exact sequence of (a) with the sequence 2.2, we see that $K^{*} \cong D / D_{\text {tors }}$, hence the result. 


\section{Limits and units of adjunctions}

Let $\mathcal{Y}$ be a class of finite $p$-groups. We shall say that $\mathcal{Y}$ is closed under taking sections if for any $R \in \mathcal{Y}$ and any section $(T, S)$ of $R$, any group isomorphic to $T / S$ belongs to $\mathcal{Y}$. In particular $\mathcal{Y}$ is closed under isomorphisms. For every $p$-group $P$, we define $\mathcal{Y}(P)$ to be the set of all sections $(T, S)$ of $P$ such that $T / S \in \mathcal{Y}$.

Let $\mathcal{Y}$ be a class of finite $p$-groups, closed under taking sections. Let $\mathcal{C}_{\mathcal{Y}}$ be the full subcategory of the category $\mathcal{C}_{p}$ whose objects are in $\mathcal{Y}$. A biset functor on $\mathcal{Y}$ is an additive functor from $\mathcal{C}_{\mathcal{Y}}$ to the category of abelian groups. Let $\mathcal{F}$ be the category of biset functors (defined on the whole of $\mathcal{C}_{p}$ ) and let $\mathcal{F}_{\mathcal{Y}}$ be the category of biset functors on $\mathcal{Y}$. Both are abelian categories. There is an obvious forgetful functor

$$
\mathcal{O}_{\mathcal{Y}}: \mathcal{F} \longrightarrow \mathcal{F}_{\mathcal{Y}}
$$

and we wish to construct functors in the opposite direction. If $M$ is a biset functor on $\mathcal{Y}$, we define, for every $p$-group $P$,

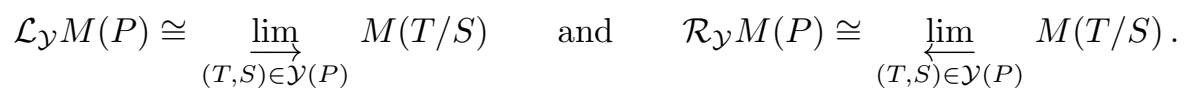

In the first case, the direct limit is constructed with respect to all induction-inflation maps and all conjugation maps. In the second case, the inverse limit is constructed with respect to all deflation-restriction maps and all conjugation maps (see (6.6) for details). For practical purposes, we always view the inverse limit $\lim _{\longleftarrow} M(T / S)$ as a subset of the direct product $\prod_{(T, S) \in \mathcal{Y}(P)} M(T / S)$.

$$
\lim _{(T, S) \in \mathcal{Y}}(P)
$$

It turns out that it is possible to put a structure of biset functors on $\mathcal{L}_{\mathcal{Y}} M$ and $\mathcal{R}_{\mathcal{Y}} M$. More precisely, we have the following theorem.

4.1. Theorem. Let $\mathcal{Y}$ be a class of finite p-groups, closed under taking sections.

(a) For every biset functor $M$ defined on $\mathcal{Y}$, there is a biset functor $\mathcal{L}_{\mathcal{Y}} M$ defined on all p-groups such that, for every p-group $P$, the evaluation of $\mathcal{L}_{\mathcal{Y}} M$ at $P$ is given by the direct limit above. Moreover, the assignment $M \mapsto \mathcal{L}_{\mathcal{Y}} M$ defines a functor $\mathcal{F}_{\mathcal{Y}} \longrightarrow \mathcal{F}$ which is left adjoint to the forgetful functor $\mathcal{O}_{\mathcal{Y}}$.

(b) For every biset functor $M$ defined on $\mathcal{Y}$, there is a biset functor $\mathcal{R}_{\mathcal{Y}} M$ defined on all p-groups such that, for every p-group $P$, the evaluation of $\mathcal{R}_{\mathcal{Y}} M$ at $P$ is given by the inverse limit above. Moreover, the assignment $M \mapsto \mathcal{R}_{\mathcal{Y}} M$ defines a functor $\mathcal{F}_{\mathcal{Y}} \longrightarrow \mathcal{F}$ which is right adjoint to the forgetful functor $\mathcal{O}_{\mathcal{Y}}$.

The proof is rather technical and is independent of the main purpose of this paper. It is included in the appendix. Because of the adjunction, there is a unit natural transformation

$$
\eta^{\mathcal{Y}}: \operatorname{id}_{\mathcal{F}} \longrightarrow \mathcal{R}_{\mathcal{Y}} \mathcal{O}_{\mathcal{Y}}
$$

and we write its evaluation at a biset functor $F$ as follows :

$$
\eta_{F}^{\mathcal{Y}}: F \longrightarrow \mathcal{R}_{\mathcal{Y}} \mathcal{O}_{\mathcal{Y}} F
$$

It is also proved in the appendix (Corollary 6.17) that when $\eta_{F}^{\mathcal{Y}}$ is evaluated at a p-group $P$, one gets a map which coincides with the product of deflation-restrictions to $\mathcal{Y}(P)$ :

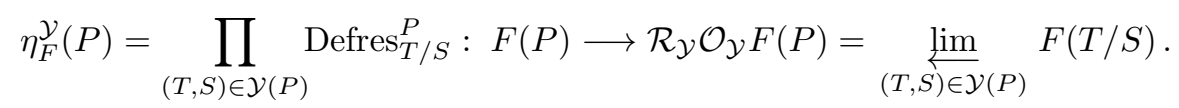


The discussion of this map is at the heart of the present paper.

We shall need to compare the constructions corresponding to two different classes of $p$-groups $\mathcal{Y}$ and $\mathcal{Z}$ such that $\mathcal{Z} \subseteq \mathcal{Y}$. In that case, for every $p$-group $P$, there is a natural homomorphism

$$
\pi_{\mathcal{Z}}^{\mathcal{Y}}(P): \mathcal{R}_{\mathcal{Y}} \mathcal{O}_{\mathcal{Y}} F(P) \longrightarrow \mathcal{R}_{\mathcal{Z}} \mathcal{O}_{\mathcal{Z}} F(P)
$$

which is simply the restriction of the projection

$$
\prod_{(T, S) \in \mathcal{Y}(P)} F(T / S) \longrightarrow \prod_{(T, S) \in \mathcal{Z}(P)} F(T / S) .
$$

It can be shown that this defines a morphism of functors $\pi_{\mathcal{Z}}^{\mathcal{Y}}: \mathcal{R}_{\mathcal{Y}} \mathcal{O}_{\mathcal{Y}} F \longrightarrow \mathcal{R}_{\mathcal{Z}} \mathcal{O}_{\mathcal{Z}} F$, but we actually only need here the evaluation $\pi_{\mathcal{Z}}^{\mathcal{Y}}(P)$ at $P$.

Our first lemma is concerned with the question of passing from a class $\mathcal{Z}$ to a larger class $\mathcal{Y}$.

4.2. Lemma. Let $\mathcal{Y}$ and $\mathcal{Z}$ be two classes of p-groups, closed under taking sections, such that $\mathcal{Z} \subseteq \mathcal{Y}$. Let $F$ be a biset functor on p-groups.

(a) If the unit morphism $\eta_{F}^{\mathcal{Z}}: F \rightarrow \mathcal{R}_{\mathcal{Z}} \mathcal{O}_{\mathcal{Z}} F$ is injective, then so are the unit morphism $\eta_{F}^{\mathcal{Y}}: F \rightarrow \mathcal{R}_{\mathcal{Y}} \mathcal{O}_{\mathcal{Y}} F$ and the morphism $\pi_{\mathcal{Z}}^{\mathcal{Y}}: \mathcal{R}_{\mathcal{Y}} \mathcal{O}_{\mathcal{Y}} F \rightarrow \mathcal{R}_{\mathcal{Z}} \mathcal{O}_{\mathcal{Z}} F$.

(b) If the unit morphism $\eta_{F}^{\mathcal{Z}}: F \rightarrow \mathcal{R}_{\mathcal{Z}} \mathcal{O}_{\mathcal{Z}} F$ is an isomorphism, then so are the unit morphism $\eta_{F}^{\mathcal{Y}}: F \rightarrow \mathcal{R}_{\mathcal{Y}} \mathcal{O}_{\mathcal{Y}} F$ and the morphism $\pi_{\mathcal{Z}}^{\mathcal{Y}}: \mathcal{R}_{\mathcal{Y}} \mathcal{O}_{\mathcal{Y}} F \rightarrow \mathcal{R}_{\mathcal{Z}} \mathcal{O}_{\mathcal{Z}} F$.

Proof. (a) Since $\mathcal{Z} \subseteq \mathcal{Y}$, for any $p$-group $P$, we have a commutative diagram

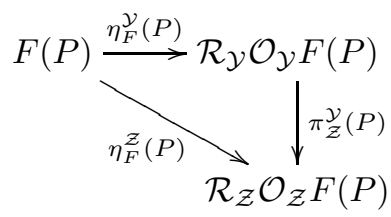

which shows that $\eta_{F}^{\mathcal{Y}}$ is injective if $\eta_{F}^{\mathcal{Z}}$ is. Moreover if $u \in \operatorname{Ker} \pi_{\mathcal{Z}}^{\mathcal{Y}}(P)$, view $u$ as a sequence $\left(u_{T, S}\right)_{(T, S) \in \mathcal{Y}(P)}$, with some compatibility conditions. Fix $(T, S)$ in $\mathcal{Y}(P)$, and consider $\left(T^{\prime} / S, S^{\prime} / S\right) \in \mathcal{Z}(T / S)$, or equivalently $\left(T^{\prime}, S^{\prime}\right) \in \mathcal{Z}(P)$ with $S \leq S^{\prime} \leq T^{\prime} \leq T$. Then Defres $T_{T^{\prime} / S^{\prime}}^{T / S} u_{T, S}=u_{T^{\prime}, S^{\prime}}=0$ and this holds for every section in $\mathcal{Z}(T / S)$. It follows that $u_{T, S}$ is in the kernel of the map

$$
\eta_{F}^{\mathcal{Z}}(T / S): F(T / S) \rightarrow \mathcal{R}_{\mathcal{Z}} \mathcal{O}_{\mathcal{Z}} F(T / S)
$$

Thus $u_{T, S}=0$, since $\eta_{F}^{\mathcal{Z}}$ is injective by assumption. So $\pi_{\mathcal{Z}}^{\mathcal{Y}}(P)$ is injective.

(b) If $\eta_{F}^{\mathcal{Z}}(P)$ is an isomorphism, then the diagram above shows that $\pi_{\mathcal{Z}}^{\mathcal{Y}}(P)$ is surjective. Hence by (a), it is an isomorphism. It now follows that $\eta_{F}^{\mathcal{y}}(P)$ is also an isomorphism.

The next lemma is concerned with the question of passing from a class $\mathcal{Y}$ to a smaller class $\mathcal{Z}$, provided a suitable assumption holds. 
4.3. Lemma. Let $\mathcal{Y}$ and $\mathcal{Z}$ be two classes of p-groups, closed under taking sections, such that $\mathcal{Z} \subseteq \mathcal{Y}$. Let $F$ be a biset functor on p-groups. If the unit morphism $\eta_{F}^{\mathcal{Y}}: F \rightarrow$ $\mathcal{R}_{\mathcal{Y}} \mathcal{O}_{\mathcal{Y}} F$ is an isomorphism and if, for every $Q \in \mathcal{Y}$, the evaluation at $Q$ of the unit morphism $\eta_{F}^{\mathcal{Z}}(Q): F(Q) \rightarrow \mathcal{R}_{\mathcal{Z}} \mathcal{O}_{\mathcal{Z}} F(Q)$ is an isomorphism, then the unit morphism $\eta_{F}^{\mathcal{Z}}: F \rightarrow \mathcal{R}_{\mathcal{Z}} \mathcal{O}_{\mathcal{Z}} F$ is an isomorphism.

Proof. For any $Q \in \mathcal{Y}$, we have a commutative diagram

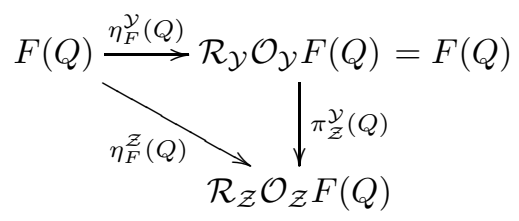

and the top map is the identity. It follows that $\pi_{\mathcal{Z}}^{\mathcal{Y}}(Q)$ can be identified with $\eta_{F}^{\mathcal{Z}}(Q)$ and is in particular an isomorphism. Now for any $p$-group $P$ and any section $(V, U)$ of $P$ such that $V / U \in \mathcal{Y}$, we have a commutative diagram

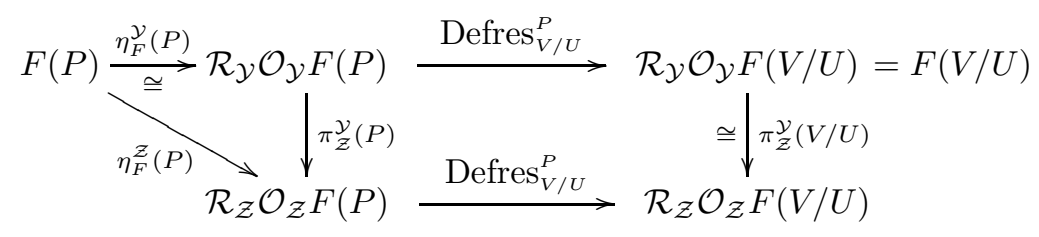

and we note that the map $\operatorname{Defres}_{V / U}^{P}$ on the first line is actually the projection on $F(V / U)$ when the limit $\mathcal{R}_{\mathcal{Y}} \mathcal{O}_{\mathcal{Y}} F(P)$ is viewed as a subset of the product $\prod_{(T, S) \in \mathcal{Y}(P)} F(T / S)$ (see Example 6.10).

When $(V, U)$ runs through the set $\mathcal{Y}(P)$ of all sections of $P$ belonging to $\mathcal{Y}$, the right hand side of the diagram gives rise to the following commutative square:

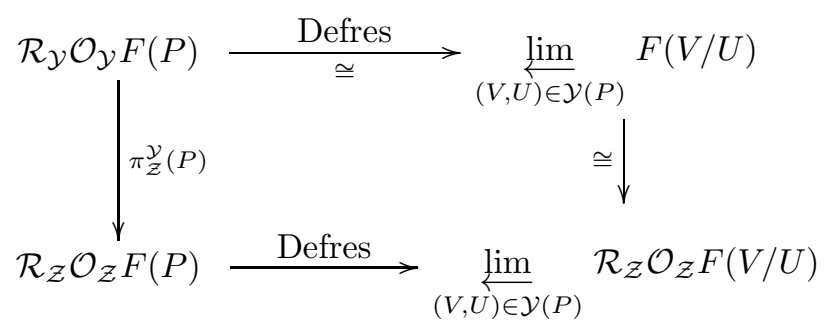

We want to prove that $\pi_{\mathcal{Z}}^{\mathcal{Y}}(P)$ is an isomorphism. The map on the right hand side is an isomorphism by the argument above. The top map Defres is an isomorphism by the very definition of $\mathcal{R}_{\mathcal{Y}} \mathcal{O}_{\mathcal{Y}} F(P)$. Thus it suffices to prove that the bottom map Defres is injective.

Let $a \in \mathcal{R}_{\mathcal{Z}} \mathcal{O}_{\mathcal{Z}} F(P)$ be in the kernel of Defres and write $a=\left(a_{T, S}\right)$ where $(T, S)$ runs through $\mathcal{Z}(P)$ and $a_{T, S} \in F(T / S)$. Since $\mathcal{Z} \subseteq \mathcal{Y}$, we can choose the component $(V, U)=$ $(T, S)$ in the right hand side limit and we have $\mathcal{R}_{\mathcal{Z}} \mathcal{O}_{\mathcal{Z}} F(T / S)=F(T / S)$, because $T / S \in \mathcal{Z}$. Then the $(T, S)$-component of $\operatorname{Defres}(a)$ is just $a_{T, S}$ (see Example 6.10 applied to the class $\mathcal{Z}$ ). Since $\operatorname{Defres}(a)=0$, we have $a_{T, S}=0$, that is, $a=0$. This proves the desired injectivity, hence $\pi_{\mathcal{Z}}^{\mathcal{Y}}(P)$ is an isomorphism. 
Returning to the previous diagram, we now deduce that $\eta_{F}^{\mathcal{Z}}(P)$ is an isomorphism, as was to be shown.

For the class of elementary abelian $p$-groups, we have the following key result.

4.4. Theorem. Let $\mathcal{E}$ be the class of elementary abelian p-groups. Let $F$ be a biset functor on p-groups and let $\eta_{F}^{\mathcal{E}}: F \rightarrow \mathcal{R}_{\mathcal{E}} \mathcal{O}_{\mathcal{E}} F$ be the unit morphism. For any fixed p-group $P$, there is a group homomorphism

$$
\sigma_{P}: \mathcal{R}_{\mathcal{E}} \mathcal{O}_{\mathcal{E}} F(P) \rightarrow F(P)
$$

such that $\eta_{F}^{\mathcal{E}}(P) \circ \sigma_{P}=|P| \cdot \mathrm{id}$.

Proof. This is exactly Theorem 5.1 in BoTh2]. Actually the homomorphism $\sigma_{P}$ is explicitly defined by the formula

$$
\sigma_{P}(u)=\sum_{(T, S) \in \mathcal{E}(P)}|S| \mu(S, T) \operatorname{Indinf}_{T / S}^{P} u_{S, \Phi(T)}
$$

for $u \in \mathcal{R}_{\mathcal{E}} \mathcal{O}_{\mathcal{E}} F(P)$, where $\mu(S, T)$ is the Möbius functions of the poset of subgroups of $P$. The proof consists in a computation which shows that $\eta_{F}^{\mathcal{E}}(P) \circ \sigma_{P}=|P| \cdot$ id.

4.5. Corollary. Let $\mathcal{Y}$ be a class of p-groups, closed under taking sections and containing the class $\mathcal{E}$ of all elementary abelian p-groups. Let $F$ be a biset functor on p-groups such that the unit morphism $\eta_{F}^{\mathcal{E}}$ is injective. For any fixed $p$-group $P$, there is a group homomorphism

$$
\tau_{P}: \mathcal{R}_{\mathcal{Y}} \mathcal{O}_{\mathcal{Y}} F(P) \rightarrow F(P)
$$

such that $\eta_{F}^{\mathcal{Y}}(P) \circ \tau_{P}=|P| \cdot$ id. In particular the cokernel of $\eta_{F}^{\mathcal{Y}}(P)$ is a $|P|$-torsion group.

Proof. Since $\mathcal{Y} \supseteq \mathcal{E}$, we have a commutative diagram

$$
F(P) \stackrel{\eta_{F}^{\mathcal{Y}}(P)}{\longrightarrow} \mathcal{R}_{\mathcal{Y}} \mathcal{O}_{\mathcal{Y}} F(P)
$$

as in the proof of Lemma 4.2. By Theorem 4.4, there exists a map

$$
\sigma_{P}: \mathcal{R}_{\mathcal{E}} \mathcal{O}_{\mathcal{E}} F(P) \rightarrow F(P)
$$

such that $\eta_{F}^{\mathcal{E}}(P) \circ \sigma_{P}=|P| \cdot$ id. Setting $\tau_{P}=\sigma_{P} \circ \pi_{\mathcal{E}}^{\mathcal{Y}}(P)$, we have

$$
\begin{aligned}
\pi_{\mathcal{E}}^{\mathcal{Y}}(P) \circ \eta_{F}^{\mathcal{Y}}(P) \circ \tau_{P} & =\pi_{\mathcal{E}}^{\mathcal{Y}}(P) \circ \eta_{F}^{\mathcal{Y}}(P) \circ \sigma_{P} \circ \pi_{\mathcal{E}}^{\mathcal{Y}}(P)=\eta_{F}^{\mathcal{E}}(P) \circ \sigma_{P} \circ \pi_{\mathcal{E}}^{\mathcal{Y}}(P) \\
& =(|P| \cdot \mathrm{id}) \circ \pi_{\mathcal{E}}^{\mathcal{Y}}(P)=\pi_{\mathcal{E}}^{\mathcal{Y}}(P) \circ(|P| \cdot \mathrm{id}) .
\end{aligned}
$$

But by Lemma 4.2. the map $\pi_{\mathcal{E}}^{\mathcal{Y}}(P)$ is injective, because we have assumed that the unit morphism $\eta_{F}^{\mathcal{E}}$ is injective. It follows that $\eta_{F}^{\mathcal{Y}}(P) \circ \tau_{P}=|P| \cdot \mathrm{id}$, as was to be shown. 


\section{The main result}

We now return to the Dade functor and prove the main result. We first work with the class $\mathcal{X}$ consisting of all elementary abelian $p$-groups and extraspecial $p$-groups of order $p^{3}$ and exponent $p$ (where $p$ is odd). By Theorem 3.3 , there is an exact sequence of biset functors

$$
0 \longrightarrow D_{\text {tors }} \longrightarrow D \longrightarrow K^{*} \longrightarrow 0
$$

and we consider $D_{\text {tors }}$ and $K^{*}$ seperately.

5.1. Proposition. The unit homomorphism

$$
\eta_{K^{*}}^{\mathcal{X}}: K^{*} \longrightarrow \mathcal{R}_{\mathcal{X}} \mathcal{O}_{\mathcal{X}} K^{*}
$$

is an isomorphism.

Proof. Consider the counit morphisms $\varepsilon_{K}^{\mathcal{X}}: \mathcal{L}_{\mathcal{X}} \mathcal{O}_{\mathcal{X}} K \rightarrow K$. By construction of $\mathcal{L}_{\mathcal{X}}$ and by a result which is dual to Corollary 6.17, for any $p$-group $P$, the image of $\varepsilon_{K}^{\mathcal{X}}(P)$ is equal to the sum of all $\operatorname{Indinf}_{T / S}^{P} K(T / S)$, where $(T, S) \in \mathcal{X}(P)$. Since $\mathcal{X}$ contains $\mathcal{X}_{2}$, this sum is the whole of $K(P)$, by Theorem 2.1. It is for this crucial result that we need to include the extraspecial group of order $p^{3}$ and exponent $p$ in our class $\mathcal{X}$. It follows that the morphism $\varepsilon_{K}^{\mathcal{X}}$ is surjective.

Let $M$ denote the kernel of $\varepsilon_{K}^{\mathcal{X}}$, so that we have a short exact sequence of functors

$$
0 \longrightarrow M \longrightarrow \mathcal{L}_{\mathcal{X}} \mathcal{O}_{\mathcal{X}} K \longrightarrow K \longrightarrow 0 \text {. }
$$

Since $B(P)$ is a free abelian group for any p-group $P$, so is its subgroup $K(P)$. Therefore, taking $\mathbb{Z}$-duals of this exact sequence gives the following short exact sequence of biset functors

$$
0 \longrightarrow K^{*} \longrightarrow\left(\mathcal{L}_{\mathcal{X}} \mathcal{O}_{\mathcal{X}} K\right)^{*} \longrightarrow M^{*} \longrightarrow 0
$$

Now the dual of a colimit is isomorphic to the limit of the duals. So there is an isomorphism

$$
\left(\mathcal{L}_{\mathcal{X}} \mathcal{O}_{\mathcal{X}} K\right)^{*} \cong \mathcal{R}_{\mathcal{X}} \mathcal{O}_{\mathcal{X}} K^{*}
$$

and the previous sequence becomes

$$
0 \longrightarrow K^{*} \stackrel{\eta_{K}^{\mathcal{X}}}{\longrightarrow} \mathcal{R}_{\mathcal{X}} \mathcal{O}_{\mathcal{X}} K^{*} \longrightarrow M^{*} \longrightarrow 0
$$

Thus for any $p$-group $P$, we have a short exact sequence of abelian groups

$$
0 \longrightarrow K^{*}(P) \stackrel{\eta_{K^{*}}^{\mathcal{X}}(P)}{\longrightarrow} \mathcal{R}_{\mathcal{X}} \mathcal{O}_{\mathcal{X}} K^{*}(P) \longrightarrow M^{*}(P) \longrightarrow 0
$$

Moreover the $\mathbb{Z}$-dual of any abelian group is torsion free, so $M^{*}(P)$ is torsion free. We are going to show that the morphism $\eta_{K^{*}}^{\mathcal{E}}$ is injective. It follows that we can apply Corollary 4.5 and therefore $M^{*}(P)$ is a $|P|$-torsion group. Thus $M^{*}(P)=0$, and the $\operatorname{map} \eta_{K^{*}}^{\mathcal{X}}$ is an isomorphism, as was to be shown.

It remains to prove the claim, i.e. to show that $\eta_{K^{*}}^{\mathcal{E}}(P)$ is injective for any $p$ group $P$. Let $\varphi \in K^{*}(P)$ such that $\eta_{K^{*}}^{\mathcal{E}}(P)(\varphi)=0$. By Corollary 6.17, this means 
that $\operatorname{Defres}_{T / S}^{P} \varphi=0$, for any section $(T, S)$ of $P$ such that $T / S$ is elementary abelian. By definition of dual functors (see the end of Section 2), this is equivalent to

$$
\varphi\left(\operatorname{Indinf}_{T / S}^{P} K(T / S)\right)=0,
$$

for any $(T, S) \in \mathcal{E}(P)$. By Theorem 2.1, we obtain $\varphi\left(K_{\varepsilon}(P)\right)=0$. Hence $p \varphi(K(P))=0$, because $p K(P) \subseteq K_{\varepsilon}(P)$, by Theorem 2.1 again. Since $\varphi$ has values in $\mathbb{Z}$, it follows that $\varphi=0$, proving the injectivity of $\eta_{K^{*}}^{\mathcal{E}}(P)$.

5.2. Remark. The functor $M$ which appears in the proof is a torsion functor, because it has the property that $M^{*}=0$. We do not know if $M=0$ or if $M$ carries some relevant information.

Now we turn to the analysis of the functor $D_{\text {tors }}$.

5.3. Proposition. Let $\mathcal{Y}$ be a class of p-groups, closed under taking subquotients, and containing $\mathcal{E}$. If $p$ is odd, the unit homomorphism

$$
\eta_{D_{\text {tors }}}^{\mathcal{Y}}: D_{\text {tors }} \longrightarrow \mathcal{R}_{\mathcal{Y}} \mathcal{O}_{\mathcal{Y}} D_{\text {tors }}
$$

is an isomorphism.

Proof. By Lemma 4.2, it suffices to consider the case $\mathcal{Y}=\mathcal{E}$. By Theorem 3.1, the morphism $\eta_{D_{\text {tors }}}^{\mathcal{E}}: D_{\text {tors }} \longrightarrow \mathcal{R}_{\mathcal{E}} \mathcal{O}_{\mathcal{E}} D_{\text {tors }}$ is injective. Moreover, by Corollary 4.5] for any $p$-group $P$, the cokernel of $\eta_{D_{\text {tors }}}^{\mathcal{E}}(P)$ is a $|P|$-torsion group. But the torsion part of the Dade group of an odd order $p$-group is a 2-torsion group (this is a consequence of Theorem 3.1, see Corollary 13.2 of [CaTh1]). It follows that the cokernel of $\eta_{D_{\text {tors }}}^{\mathcal{E}}(P)$ is trivial. Thus $\eta_{D_{\text {tors }}}^{\mathcal{E}}$ is an isomorphism.

5.4. Remark. This proposition can be improved, see Remark 5.8 .

Putting together the previous two propositions, we obtain the following weak form of our main result.

5.5. Theorem. Let $p$ be an odd prime number. Let $\mathcal{X}$ be the class of p-groups consisting of all elementary abelian p-groups and the groups isomorphic to the extraspecial group of order $p^{3}$ and exponent $p$. Then the unit morphism

$$
\eta_{D}^{\mathcal{X}}: D \rightarrow \mathcal{R}_{\mathcal{X}} \mathcal{O}_{\mathcal{X}} D
$$

is an isomorphism.

Proof. We apply the functor $\mathcal{R}_{\mathcal{X}} \mathcal{O}_{\mathcal{X}}$ to the exact sequence

$$
0 \longrightarrow D_{\text {tors }} \longrightarrow D \longrightarrow K^{*} \longrightarrow 0
$$

of Theorem 3.3. Since this functor is left exact (because $\mathcal{O}_{\mathcal{X}}$ is exact and $\mathcal{R}_{\mathcal{X}}$ has a left adjoint), the commutative diagram

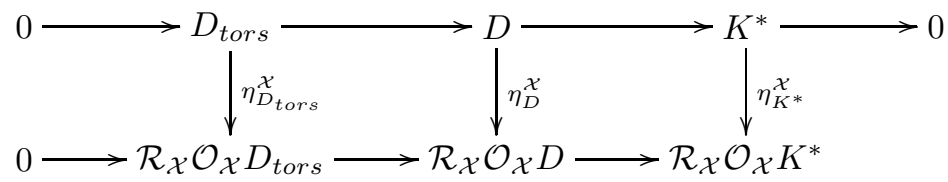


has exact rows. By Propositions 5.1 and 5.3. both vertical arrows $\eta_{D_{\text {tors }}}^{\mathcal{X}}$ and $\eta_{K^{*}}^{\mathcal{X}}$ are isomorphisms. Therefore $\eta_{D}^{\mathcal{X}}$ is an isomorphism.

In order to pass from the class $\mathcal{X}$ to the smaller class $\mathcal{X}_{3}$, we need the following result about the Dade group of elementary abelian $p$-groups.

5.6. Lemma. Let $\mathcal{E}_{3}$ be the class of all elementary abelian p-groups of rank $\leq 3$. If $H$ is an elementary abelian p-group, the map

$$
\eta_{D}^{\mathcal{E}_{3}}(H): D(H) \longrightarrow \mathcal{R}_{\mathcal{E}_{3}} \mathcal{O}_{\mathcal{E}_{3}} D(H)
$$

is an isomorphism.

Proof. By Corollary 6.17, the map $\eta_{D}^{\mathcal{E}_{3}}(H)$ coincides with the map

$$
\prod_{(T, S) \in \mathcal{E}_{3}(H)} \operatorname{Defres}_{T / S}^{H}: D(H) \longrightarrow \underbrace{\lim }_{(T, S) \in \mathcal{E}_{3}(H)} D(T / S) .
$$

We prove the result by induction on $|H|$. If $|H| \leq p^{3}$, then $\varliminf_{(T, S) \in \mathcal{E}_{3}(H)} D(T / S)=D(H)$ and there is nothing to prove. This starts induction. Assume $|H|>p^{3}$, and let $u=$ $\left(u_{T, S}\right)_{(T, S) \in \mathcal{E}_{3}(H)}$ be an element of $\varliminf_{(T, S) \in \mathcal{E}_{3}(H)}^{\lim } D(T / S)$

Fix a non trivial subgroup $J$ of $H$. The sequence of elements $u_{T, S}$, with $J \leq S \leq$ $T \leq H$ and $|T / S| \leq p^{3}$ is an element of $\lim _{(T, S) \in \mathcal{E}_{3}(H / J)} D(H / J)$. Hence by induction hypothesis, there exists a unique element $v_{J} \in D(H / J)$ such that $\operatorname{Defres}_{T / S}^{H / J} v_{J}=u_{T, S}$, for any section $(T, S)$ of $H$ with $J \leq S \leq T \leq H$ and $|T / S| \leq p^{3}$.

The uniqueness of $v_{J}$ implies that $\operatorname{Def}_{H / J^{\prime}}^{H / J} v_{J}=v_{J^{\prime}}$ whenever $J$ and $J^{\prime}$ are subgroups of $H$ with $1<J \leq J^{\prime} \leq H$. So the sequence $\left(v_{J}\right)_{1<J \leq H}$ is an element of $\underbrace{}_{1<J \leq H} D(H / J)$. Now by Lemma 2.2 of BoTh2, the deflation map

$$
D(H) \longrightarrow \underbrace{\lim }_{1<J \leq H} D(H / J)
$$

is an isomorphism. Explicitly, it was observed that the element

$$
w=-\sum_{1<J \leq H} \mu(1, J) \operatorname{Inf}_{H / J}^{H} v_{J} \in D(H)
$$

is such that $\operatorname{Def}_{H / J}^{H} w=v_{J}$ for any non trivial subgroup $J$ of $H$ (where $\mu$ denotes the Möbius function of the poset of subgroups of $H$ ). It follows that $\operatorname{Defres}_{T / S}^{H} w=u_{T, S}$ for any $(T, S) \in \mathcal{E}_{3}(H)$ with $S \neq 1$.

Let $F$ be any subgroup of $H$ of order $p^{3}$, and consider $f_{F}=\operatorname{Res}_{F}^{H} w-u_{F, 1}$. Then clearly $\operatorname{Def}_{F / F^{\prime}}^{F} f_{F}=0$ whenever $F^{\prime}$ is a non trivial subgroup of $F$. It follows that

$$
f_{F} \in \bigcap_{1<F^{\prime} \leq F} \operatorname{Ker}\left(\operatorname{Def}_{F / F^{\prime}}^{F}\right)
$$

which is the subgroup of $D(F)$ consisting of endo-trivial modules (see Lemma 1.2 in BoTh1]). Since this subgroup is infinite cyclic generated by the class $\Omega_{F}$ of the 
augmentation ideal of $k F$ (Dade's theorem, see Theorem 1.4 in BoTh1), there exists a unique integer $m_{F}$ such that $f_{F}=m_{F} \Omega_{F}$. Now if $F^{\prime}$ is another subgroup of order $p^{3}$ of $H$ such that $F \cap F^{\prime}$ has order at least $p^{2}$, we have

$$
\begin{aligned}
\operatorname{Res}_{F \cap F^{\prime}}^{F} f_{F} & =\operatorname{Res}_{F \cap F^{\prime}}^{H} w-\operatorname{Res}_{F \cap F^{\prime}}^{F} u_{F, 1} \\
& =\operatorname{Res}_{F \cap F^{\prime}}^{H} w-u_{F \cap F^{\prime}, 1} \\
& =\operatorname{Res}_{F \cap F^{\prime}}^{F^{\prime}} f_{F^{\prime}} .
\end{aligned}
$$

Thus $m_{F} \Omega_{F \cap F^{\prime}}=m_{F^{\prime}} \Omega_{F \cap F^{\prime}}$, and $m_{F}=m_{F^{\prime}}$ because $\Omega_{F \cap F^{\prime}}$ has infinite order. Thus $m_{F}=m_{F^{\prime}}$ if $\left|F \cap F^{\prime}\right| \geq p^{2}$, and since the poset of subgroups of $H$ of order $p^{2}$ and $p^{3}$ is connected, $m_{F}$ does not depend on the subgroup $F$ of order $p^{3}$. Set $m=m_{F}$, and consider the element $t=w-m \Omega_{H}$. For any section $(T, S) \in \mathcal{E}_{3}(H)$ with $S \neq 1$, we have again

$$
\operatorname{Defres}_{T / S}^{H} t=\operatorname{Defres}_{T / S}^{H} w=u_{T, S} \text {. }
$$

Moreover, if $E$ is any subgroup of $H$ of order at most $p^{3}$, choose some subgroup $F$ of order $p^{3}$ containing $E$. Then

$$
\operatorname{Defres}_{E / 1}^{H} t=\operatorname{Res}_{E}^{H} t=\operatorname{Res}_{E}^{F} \operatorname{Res}_{F}^{H} t=\operatorname{Res}_{E}^{F} u_{F, 1}=u_{E, 1} .
$$

It follows that $\eta_{D}^{\mathcal{E}_{3}}(H)(t)=u$, and so $\eta_{D}^{\mathcal{E}_{3}}(H)$ is surjective. It is also injective by Theorem 3.1 and this completes the proof of the lemma.

We have now paved the way for the final version of our main result.

5.7. Theorem. Let $p$ be an odd prime number and let $\mathcal{X}_{3}$ be the class of p-groups consisting of all elementary abelian p-groups of rank $\leq 3$ and the groups isomorphic to the extraspecial group of order $p^{3}$ and exponent $p$. Then the unit morphism

$$
\eta_{D}^{\mathcal{X}_{3}}: D \rightarrow \mathcal{R}_{\mathcal{X}_{3}} \mathcal{O}_{\mathcal{X}_{3}} D
$$

is an isomorphism. In other words, if $P$ is a p-group, then the map

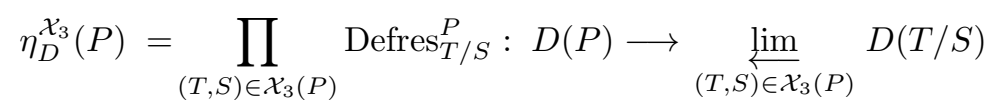

is a group isomorphism.

Proof. We are going to apply Lemma 4.3 to the class $\mathcal{X}$ and the subclass $\mathcal{X}_{3}$. First note that the unit morphism $\eta_{D}^{\mathcal{X}}$ is an isomorphism by Theorem 5.5. Moreover, we must show that, for any $Q \in \mathcal{X}$, the unit morphism $\eta_{D}^{\mathcal{X}_{3}}(Q)$ evaluated at $Q$ is an isomorphism. This is obvious if $Q \in \mathcal{X}_{3}$ (in that case $\eta_{D}^{\mathcal{X}_{3}}(Q)$ is the identity). Now if $Q \in \mathcal{X}-\mathcal{X}_{3}$, then $Q$ is elementary abelian of rank $\geq 4$ and the family $\mathcal{X}_{3}(Q)$ coincides with $\mathcal{E}_{3}(Q)$. Thus $\eta_{D}^{\mathcal{X}_{3}}(Q)=\eta_{D}^{\mathcal{E}_{3}}(Q)$ and this is an isomorphism by Lemma 5.6. Thus the assumptions of Lemma 4.3 are satisfied and therefore $\eta_{D}^{\mathcal{X}_{3}}$ is an isomorphism.

5.8. Remark. Proposition 5.3 can be improved in the same way, replacing the class $\mathcal{E}$ by the smaller class $\mathcal{E}_{3}$. Thus we obtain that, if $p$ is odd, the unit homomorphism

$$
\eta_{D_{\text {tors }}}^{\mathcal{E}_{3}}: D_{\text {tors }} \longrightarrow \mathcal{R}_{\mathcal{E}_{3}} \mathcal{O}_{\mathcal{E}_{3}} D_{\text {tors }}
$$

is an isomorphism. 
5.9. Remark. The class $\mathcal{X}_{3}$ is exactly the class of $p$-groups of order at most $p^{3}$ and exponent dividing $p$.

5.10. Remark. Theorem 5.7 holds more generally for any class of $p$-groups containing $\mathcal{X}_{3}$. This follows immediately from Lemma 4.2.

5.11. Remark. Theorem 5.7 does not hold if we remove from $\mathcal{X}_{3}$ the groups which are isomorphic to one of the two groups of order $p^{3}$ (elementary abelian or extraspecial). In order to prove this, we let $P \in \mathcal{X}_{3}$ with $|P|=p^{3}$, we consider the unit morphism $\eta_{D}^{\mathcal{E}_{2}}(P)$ corresponding to proper sections of $P$ and we show that $\eta_{D}^{\mathcal{E}_{2}}(P)$ is not surjective. Note first that $P$ has more than one elementary abelian subgroup of rank 2 , and that each of them is normal in $P$. Let $Q$ be any of them and define, for any $(T, S) \in \mathcal{E}_{2}(P)$,

$$
u_{T, S}= \begin{cases}2 \Omega_{Q} & \text { if }(T, S)=(Q, 1) \\ 0 & \text { otherwise }\end{cases}
$$

The family $u=\left(u_{T, S}\right)$ defines an element of $\lim _{(T, S) \in \mathcal{E}_{2}(P)} D(T / S)$ because $\operatorname{Res}_{R}^{Q}\left(2 \Omega_{Q}\right)=$ $2 \Omega_{R}=0$ whenever $R<Q$ (because $D(R)=\mathbb{Z} / 2 \mathbb{Z}$ if $|R|=p$ ). We want to prove that $u$ is not in the image of $\eta_{D}^{\mathcal{E}_{2}}(P)$. Suppose there exists $v \in D(P)$ whose image is $u$. Then any section $(T, S)$ of $P$ with $S \neq 1$ belongs to $\mathcal{E}_{2}(P)$ and $\operatorname{Defres}_{T / S}^{P}(v)=u_{T, S}=0$ by definition of $u$. It follows that $v$ belongs to the subgroup $T(P)$ of $D(P)$ consisting of classes of endo-trivial modules. Moreover $\operatorname{Res}_{Q}^{P}(v)=2 \Omega_{Q}$ and $\operatorname{Res}_{Q^{\prime}}^{P}(v)=0$ for any other elementary abelian subgroup $Q^{\prime}$ of rank 2 . This is impossible if $P$ is elementary abelian of rank 3 because $T(P)=\mathbb{Z}$ generated by $\Omega_{P}$ and we would obtain $v=2 \Omega_{P}$ (because $\operatorname{Res}_{Q}^{P}(v)=2 \Omega_{Q}$ ) and also $v=0$ (because $\operatorname{Res}_{Q^{\prime}}^{P}(v)=0$ ). This is also impossible if $P$ is extraspecial of order $p^{3}$ and exponent $p$ because the condition $\operatorname{Res}_{Q^{\prime}}^{P}(v)=0$ for any $Q^{\prime} \neq Q$ implies that $\operatorname{Res}_{Q}^{P}(v)$ must be a multiple of $2 p \Omega_{Q}$, using the description of $T(P)$ given in CaTh2] (see Theorems 3.1 and 6.1 of that paper).

\section{Appendix}

The purpose of this appendix is to provide a proof of Theorem 4.1 We shall only prove the statement about $\mathcal{R}_{\mathcal{Y}}$ because the treatment of $\mathcal{L}_{\mathcal{Y}}$ is similar. This type of construction of adjoints is more or less standard in category theory (see for instance [GZ]). We provide here an explicit treatment in our situation.

We first start with technical lemmas about bisets, using the following notation.

6.1. Notation. Let $P$ and $Q$ be two groups.

(a) If $V$ is a right $Q$-set and $U$ is a left $Q$-set, $\left(v,_{Q} u\right)$ denotes the image in $V \times{ }_{Q} U$ of the pair $(v, u)$ of $V \times U$.

(b) Let $U$ be a $(Q, P)$-biset. If $S$ is a subgroup of $P$ and $u$ is an element of $U$, set

$$
{ }^{u} S=\{y \in Q \mid \exists s \in S, u s=y u\} .
$$

Then ${ }^{u} S$ is a subgroup of $Q$. Similarly, if $T$ is a subgroup of $Q$, set

$$
T^{u}=\{x \in P \mid \exists t \in T, t u=u x\} .
$$

It is a subgroup of $P$. 
6.2. Remark. The notation in (b) extends the standard notation for conjugation in the following sense. Let $P$ be a $p$-group and $S$ a subgroup of $P$.

(1) If $P$ is a subgroup of $Q$ and $U=P$ viewed as a $(Q, P)$-biset, then ${ }^{u} S=u S u^{-1}$.

(2) If $Q$ is a subgroup of $P$ and $U=P$ viewed as a $(Q, P)$-biset, then ${ }^{u} S=Q \cap u S u^{-1}$.

(3) If $Q=P / R$ is a quotient group of $P$ and $U=P / R$ viewed as a $(Q, P)$-biset, then ${ }^{u} S$ is the image in $Q$ of the conjugate subgroup $u S u^{-1}$.

(4) If $P=Q / R$ is a quotient group of $Q$ and $U=Q / R$ viewed as a $(Q, P)$-biset, then ${ }^{u} S$ is the inverse image in $Q$ of the conjugate subgroup $u S u^{-1}$.

6.3. Remark. Note that $1^{u}$ is the stabilizer of $u$ in $P$ and ${ }^{u} 1$ is the stabilizer of $u$ in $Q$. In the definition of ${ }^{u} S$, the element $s$ is unique up to left multiplication by an element of $S \cap 1^{u}$. Similarly, in the definition of $T^{u}$, the element $t$ is unique up to right multiplication by an element of $T \cap{ }^{u}$. This explains why these subgroups appear in parts (b) and (b') of the next lemma.

6.4. Lemma. Let $P, Q$ and $R$ be groups, let $U$ be a $(Q, P)$-biset, and let $V$ be an $(R, Q)$-biset. Let $u \in U$ and $v \in V$.

(a) If $T$ is a subgroup of $Q$ and if $x \in P$, then $\left(T^{u}\right)^{x}=T^{u x}$.

(a') If $X$ is a subgroup of $P$ and if $y \in Q$, then ${ }^{y}\left({ }^{u} X\right)={ }^{y u} X$.

(b) If $(T, S)$ is a section of $Q$, then $\left(T^{u}, S^{u}\right)$ is a section of $P$, and there are group isomorphisms

$$
T^{u} / S^{u} \cong\left(T \cap{ }^{u} P\right) /\left(S \cap{ }^{u} P\right)\left(T \cap{ }^{u} 1\right) \cong\left(T \cap{ }^{u} P\right) S /\left(T \cap{ }^{u} 1\right) S .
$$

In particular, the quotient $T^{u} / S^{u}$ is isomorphic to a subquotient of $T / S$.

(b') If $(Y, X)$ is a section of $P$, then $\left({ }^{u} Y,{ }^{u} X\right)$ is a section of $Q$, and there are group isomorphisms

$$
{ }^{u} Y /{ }^{u} X \cong\left(Y \cap Q^{u}\right) /\left(X \cap Q^{u}\right)\left(Y \cap 1^{u}\right) \cong\left(Y \cap Q^{u}\right) X /\left(Y \cap 1^{u}\right) X
$$

In particular, the quotient ${ }^{u} Y /{ }^{u} X$ is isomorphic to a subquotient of $Y / X$.

(c) If $X$ is a subgroup of $P$, then ${ }^{v}\left({ }^{u} X\right)={ }^{(v,}{ }_{Q}^{u)} X$.

(c') If $Z$ is a subgroup of $R$, then $\left(Z^{v}\right)^{u}=Z^{\left(v,_{Q} u\right)}$.

Proof. We only prove (a), (b), (c), because the proofs of (a'), (b'), (c') are similar.

(a) By definition,

$$
\begin{aligned}
T^{u x} & =\{g \in P \mid \exists t \in T, \text { tux }=u x g\} \\
& =\left\{g \in P \mid \exists t \in T, \text { tu }=u x g x^{-1}\right\} \\
& =\left\{g \in P \mid{ }^{x} g \in T^{u}\right\}=\left(T^{u}\right)^{x} .
\end{aligned}
$$

(b) Suppose that $(T, S)$ is a section of $Q$. Then $S^{u}$ is clearly a subgroup of $T^{u}$. Moreover if $x \in T^{u}$, then there exists $t \in T$ such that $t u=u x$. Thus

$$
\left(S^{u}\right)^{x}=S^{u x}=S^{t u}=S^{u}
$$


Here the first equality follows from (a) and the last one holds because $t \in T$ normalizes $S$. This shows that $S^{u} \unlhd T^{u}$. Consider the map

$$
T^{u} / S^{u} \longrightarrow\left(T \cap{ }^{u} P\right) /\left(S \cap{ }^{u} P\right)\left(T \cap{ }^{u} 1\right), \quad x S^{u} \mapsto t\left(S \cap{ }^{u} P\right)\left(T \cap{ }^{u} 1\right),
$$

where $t$ is any element of $T$ such that $t u=u x$. It is routine to check that it is a well defined group homomorphism. Similarly the map

$$
\left(T \cap{ }^{u} P\right) /\left(S \cap{ }^{u} P\right)\left(T \cap{ }^{u} 1\right) \longrightarrow T^{u} / S^{u}, \quad t\left(S \cap{ }^{u} P\right)\left(T \cap{ }^{u} 1\right) \mapsto x S^{u},
$$

where $x$ is any element of $P$ such that $t u=u x$, is a well defined group homomorphism. Clearly these two group homomorphisms are mutual inverse.

Now setting $N=\left(T \cap{ }^{u} 1\right) S$, we have

$$
\left(T \cap{ }^{u} P\right) S /\left(T \cap{ }^{u} 1\right) S=\left(T \cap{ }^{u} P\right) N / N \cong\left(T \cap{ }^{u} P\right) /\left(T \cap{ }^{u} P \cap N\right),
$$

and

$$
T \cap{ }^{u} P \cap N=T \cap{ }^{u} P \cap\left(T \cap{ }^{u} 1\right) S=\left(T \cap{ }^{u} 1\right)\left(T \cap{ }^{u} P \cap S\right)=\left(T \cap{ }^{u} 1\right)\left(S \cap{ }^{u} P\right) .
$$

This proves the second isomorphism.

Now the group $\left(T \cap{ }^{u} P\right) /\left(S \cap{ }^{u} P\right)\left(T \cap{ }^{u} 1\right)$ is a factor group of $\left(T \cap{ }^{u} P\right) /\left(S \cap{ }^{u} P\right)$, which is isomorphic to $\left(T \cap{ }^{u} P\right) S / S$, and this is a subgroup of $T / S$. Thus $T^{u} / S^{u}$ is isomorphic to a subquotient of $T / S$.

(c) By definition

$$
\begin{aligned}
{ }^{\left(v,{ }_{Q}{ }^{u)} X\right.} & =\left\{z \in R \mid \exists x \in X, z\left(v,{ }_{Q} u\right)=\left(v,{ }_{Q} u\right) x\right\} \\
& =\left\{z \in R \mid \exists x \in X,\left(z v,{ }_{Q} u\right)=\left(v,_{Q} u x\right)\right\} \\
& =\{z \in R \mid \exists x \in X, \exists y \in Q, z v=v y, y u=u x\} \\
& =\{z \in R \mid \exists y \in Q, \exists x \in X, z v=v y, y u=u x\} \\
& =\left\{z \in R \mid \exists y \in{ }^{u} X, z v=v y\right\} \\
& ={ }^{v}\left({ }^{u} X\right)
\end{aligned}
$$

This completes the proof.

6.5. Lemma. Let $P, Q$, and $R$ be groups, let $U$ be a $(Q, P)$-biset, and let $V$ be an $(R, Q)$-biset. Let moreover $(D, C)$ be a section of $R$, let $(B, A)$ be a section of $Q$ and assume that $A$ acts trivially on $C \backslash V$ (on the right). Then there is an isomorphism of $(D / C, P)$-bisets

$$
(C \backslash V) \times_{B}(A \backslash U) \cong C \backslash\left(V \times_{B} U\right)
$$

Proof. The maps $\left(C v,{ }_{B} A u\right) \mapsto C\left(v,_{B} u\right)$ and $C\left(v,_{B} u\right) \mapsto\left(C v,_{B} A u\right)$ are well defined, and mutual inverse biset isomorphisms.

Let $\mathcal{Y}$ be a class of finite $p$-groups, closed under taking sections. If $F$ is biset functor defined on $\mathcal{Y}$ and if $P$ is an arbitrary $p$-group, we have defined

$$
\mathcal{R}_{\mathcal{Y}} F(P)=\lim _{(T, S) \in \mathcal{Y}(P)} F(T / S)
$$


Recall that $\mathcal{Y}(P)$ is the set of sections $(T, S)$ of $P$ such that $T / S \in \mathcal{Y}$ and that the definition of the limit means that the group $\mathcal{R}_{\mathcal{Y}} F(P)$ is the set of sequences $\left(l_{T, S}\right)_{(T, S) \in \mathcal{Y}(P)}$ indexed by $\mathcal{Y}(P)$, where $l_{T, S} \in F(T / S)$, subject to the following conditions :

1. If $(T, S)$ and $\left(T^{\prime}, S^{\prime}\right)$ are elements of $\mathcal{Y}(P)$ such that $S \leq S^{\prime} \leq T^{\prime} \leq T$, then

$$
\operatorname{Defres}_{T^{\prime} / S^{\prime}}^{T / S} l_{T, S}=l_{T^{\prime}, S^{\prime}}
$$

where Defres $\begin{gathered}T / S \\ T^{\prime} / S^{\prime}\end{gathered}$ is the set $S^{\prime} \backslash T$, viewed as a $\left(T^{\prime} / S^{\prime}, T / S\right)$-biset.

2. If $(T, S) \in \mathcal{Y}(P)$ and if $x \in P$, then

$$
{ }^{x} l_{T, S}=l_{{ }^{x} T,{ }^{x} S}
$$

Now we want to introduce a structure of biset functor on $\mathcal{R}_{\mathcal{Y}} F$. Whenever $P$ and $Q$ are $p$-groups and $U$ is a finite $(Q, P)$-biset, we need to define a map

$$
\mathcal{R}_{\mathcal{Y}} F(U): \mathcal{R}_{\mathcal{Y}} F(P) \rightarrow \mathcal{R}_{\mathcal{Y}} F(Q) .
$$

If $l$ is an element of $\mathcal{R}_{\mathcal{Y}} F(P)$, and if $(T, S) \in \mathcal{Y}(Q)$, we set

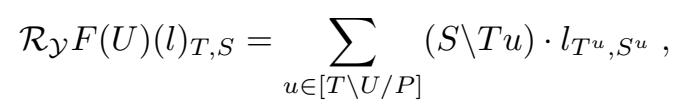

where $[T \backslash U / P]$ is any set of representatives of $(T, P)$-orbits on $U$, where $S \backslash T u$ is viewed as a $\left(T / S, T^{u} / S^{u}\right)$-biset, and $(S \backslash T u) \cdot l_{T^{u}, S^{u}}$ denotes the image of $l_{T^{u}, S^{u}}$ by this biset. This makes sense since $\left(T^{u}, S^{u}\right) \in \mathcal{Y}(P)$ if $(T, S) \in \mathcal{Y}(Q)$, by Lemma 6.4.

6.8. Remark. If we want to make this definition explicit in terms of elementary operations, we observe that it is equivalent to

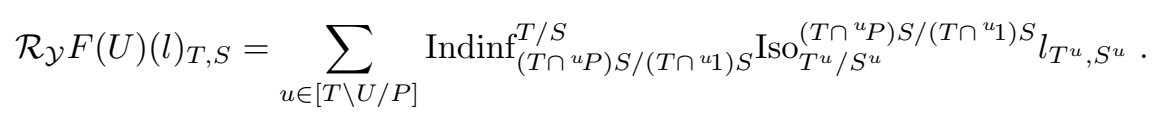

6.9. Lemma. The element $\mathcal{R}_{\mathcal{Y}} F(U)(l)_{T, S}$ is well defined, that is, it does not depend on the choice of the set of representatives $[T \backslash U / P]$.

Proof. Changing the set of representatives $[T \backslash U / P]$ amounts to replacing each element $u$ in this set by an element $u^{\prime}=t_{u} u x_{u}$, where $t_{u} \in T$ and $x_{u} \in P$. This gives a new set of representatives of orbits, denoted by $[T \backslash U / P]^{\prime}$. With this new set of representatives, the sum in (6.7) becomes

$$
\begin{aligned}
\Sigma^{\prime} & =\sum_{u^{\prime} \in[T \backslash U / P]^{\prime}}\left(S \backslash T u^{\prime}\right) \cdot l_{T^{u^{\prime}}, S^{u^{\prime}}} \\
& =\sum_{u \in[T \backslash U / P]}\left(S \backslash T u x_{u}\right) \cdot l_{\left(T^{u}\right)^{x_{u}},\left(S^{u}\right)^{x_{u}}} \\
& =\sum_{u \in[T \backslash U / P]}\left(S \backslash T u x_{u}\right) \cdot x_{u}^{-1} l_{T^{u}, S^{u}} \quad\left(\text { since } \quad l \in \mathcal{R}_{\mathcal{Y}} F(P)\right), \\
& =\sum_{u \in[T \backslash U / P]}(S \backslash T u) \cdot l_{T^{u}, S^{u}} .
\end{aligned}
$$


The latter equality follows from the fact that, for any $x \in P,(S \backslash T u) \circ \operatorname{Conj}(x) \cong S \backslash T u x$ as $\left(T / S, T^{u x} / S^{u x}\right)$-bisets (where $\operatorname{Conj}(x)$ is the $\left(T^{u} / S^{u}, T^{u x} / S^{u x}\right)$-biset $T^{u} / S^{u}$ with right action of $T^{u x} / S^{u x}$ consisting of conjugation by $x$ followed by right multiplication). The lemma follows.

6.10. Example. Let $F \in \mathcal{F}_{\mathcal{Y}}$, let $P$ be a $p$-group, let $(T, S)$ and $\left(T^{\prime}, S^{\prime}\right)$ be elements of $\mathcal{Y}(P)$ such that $S \leq S^{\prime} \leq T^{\prime} \leq T$. We view $\left(T^{\prime}, S^{\prime}\right)$ as a section of $T / S$ via the canonical isomorphism $\left(T^{\prime} / \bar{S}\right) /\left(S^{\prime} / S\right) \cong T^{\prime} / S^{\prime}$. If $l \in \mathcal{R}_{\mathcal{Y}} F(P)$, then

$$
\left(\operatorname{Defres}_{T / S}^{P} l\right)_{T^{\prime}, S^{\prime}}=l_{T^{\prime}, S^{\prime}} .
$$

Indeed in this case, the group $Q$ is equal to $T / S$ and the biset $U$ is $S \backslash P$. Hence $\left[T^{\prime} \backslash U / P\right]$ has one element, which can be chosen to be $\{S\}$, and in this case

$$
\mathcal{R}_{\mathcal{Y}} F(U)(l)_{T^{\prime}, S^{\prime}}=\left(S^{\prime} \backslash T^{\prime}\right) \cdot l_{T^{\prime}, S^{\prime}}=l_{T^{\prime}, S^{\prime}},
$$

because the $\left(T^{\prime} / S^{\prime}, T^{\prime} / S^{\prime}\right)$-biset $S^{\prime} \backslash T^{\prime}$ is the identity.

6.11. Lemma. Let $l \in \mathcal{R}_{\mathcal{Y}} F(P)$. When $(T, S)$ runs through $\mathcal{Y}(Q)$, the family $\mathcal{R}_{\mathcal{Y}} F(U)(l)_{T, S}$ defined in 6.7 is an element of $\mathcal{R}_{\mathcal{Y}} F(Q)$.

Proof. Let $(T, S)$ and $\left(T^{\prime}, S^{\prime}\right)$ be elements of $\mathcal{Y}(Q)$ such that $S \leq S^{\prime} \leq T^{\prime} \leq T$. Then $\operatorname{Defres}_{T^{\prime} / S^{\prime}}^{T / S}$ is the set $S^{\prime} \backslash T$, viewed as a $\left(T^{\prime} / S^{\prime}, T / S\right)$-biset. It follows that

$$
\begin{aligned}
\operatorname{Defres}_{T^{\prime} / S^{\prime}}^{T / S}\left(\mathcal{R}_{\mathcal{Y}} F(U)(l)_{T, S}\right) & =S^{\prime} \backslash T \times_{T / S} \sum_{u \in[T \backslash U / P]}(S \backslash T u) \cdot l_{T^{u}, S^{u}} \\
& =\sum_{u \in[T \backslash U / P]}\left(S^{\prime} \backslash T u\right) \cdot l_{T^{u}, S^{u}},
\end{aligned}
$$

because by Lemma 6.5, there is a biset isomorphism

$$
\left(S^{\prime} \backslash T\right) \times_{T / S}(S \backslash T u) \cong S^{\prime} \backslash\left(T \times_{T} T u\right)=S^{\prime} \backslash T u .
$$

Now the set of orbits of $\left(T^{\prime} / S^{\prime}\right) \times\left(T^{u} / S^{u}\right)$ on the biset $S^{\prime} \backslash T u$ is the set $T^{\prime} \backslash T u / T^{u}$, which is in bijective correspondence with the set $T^{\prime} \backslash T / T \cap{ }^{u} P$. Hence there is an isomorphism of $\left(T^{\prime} / S^{\prime}, T^{u} / S^{u}\right)$-bisets

$$
S^{\prime} \backslash T u \cong \bigsqcup_{t \in\left[T^{\prime} \backslash T / T \cap{ }^{u} P\right]} S^{\prime} \backslash T^{\prime} t u T^{u}
$$

It follows that

$$
\operatorname{Defres}_{T^{\prime} / S^{\prime}}^{T / S}\left(\mathcal{R}_{\mathcal{Y}} F(U)(l)_{T, S}\right)=\sum_{\substack{u \in[T \backslash U / P] \\ t \in\left[T^{\prime} \backslash T / T \cap{ }^{u} P\right]}} S^{\prime} \backslash T^{\prime} t u T^{u} \cdot l_{T^{u}, S^{u}} .
$$

On the other hand, we have by definition

$$
\begin{aligned}
& \mathcal{R}_{\mathcal{Y}} F(U)(l)_{T^{\prime}, S^{\prime}}=\sum_{v \in\left[T^{\prime} \backslash U / P\right]}\left(S^{\prime} \backslash T^{\prime} v\right) \cdot l_{T^{\prime v}, S^{\prime} v} \\
& =\sum_{\substack{u \in[T \backslash U / P] \\
t \in\left[T^{\prime} \backslash T / T \cap{ }^{u} P\right]}}\left(S^{\prime} \backslash T^{\prime} t u\right) \cdot l_{T^{\prime t u}, S^{\prime t u}}
\end{aligned}
$$


Now $S^{u} \leq S^{\prime t u} \leq T^{\prime t u} \leq T^{u}$, thus by Example 6.10

$$
l_{T^{\prime t u}, S^{\prime t u}}=\operatorname{Defres}_{T^{\prime t u} / S^{\prime t u}}^{T^{u} / S^{u}} l_{T^{u}, S^{u}}=S^{\prime t u} \backslash T^{u} \cdot l_{T^{u}, S^{u}} .
$$

It follows that

$$
\mathcal{R}_{\mathcal{Y}} F(U)(l)_{T^{\prime}, S^{\prime}}=\sum_{\substack{u \in[T \backslash U / P] \\ t \in\left[T^{\prime} \backslash T / T \cap{ }^{u} P\right]}}\left(S^{\prime} \backslash T^{\prime} t u\right) \times_{T^{\prime t u} / S^{\prime t u}}\left(S^{\prime t u} \backslash T^{u}\right) \cdot l_{T^{u}, S^{u}} .
$$

We have to prove that this sum coincides with the expression in (6.12). By Lemma 6.5, for any $u \in U$ and any $t \in T$, there is an isomorphism of $\left(T^{\prime} / S^{\prime}, T^{u} / S^{u}\right)$-bisets

$$
\left(S^{\prime} \backslash T^{\prime} t u\right) \times_{T^{\prime t u} / S^{\prime t u}}\left(S^{\prime t u} \backslash T^{u}\right) \cong S^{\prime} \backslash\left(T^{\prime} t u \times_{T^{\prime t u}} T^{u}\right) .
$$

But it is easy to see that $T^{\prime} t u \times_{T^{\prime t u}} T^{u} \cong T^{\prime} t u T^{u}$ as $\left(T^{\prime}, T^{u}\right)$-bisets, so that we obtain $S^{\prime} \backslash\left(T^{\prime} t u \times_{T^{\prime t u}} T^{u}\right) \cong S^{\prime} \backslash T^{\prime} t u\left(T^{u}\right)$.

6.13. Lemma. Let $P, Q$, and $R$ be finite p-groups.

(a) Let $U$ be the set $P$, viewed as a $(P, P)$-biset by left and right multiplication. Then $\mathcal{R}_{\mathcal{Y}} F(U)$ is the identity map.

(b) Let $U$ be a $(Q, P)$-biset and let $V$ be an $(R, Q)$-biset. Then

$$
\mathcal{R}_{\mathcal{Y}} F(V) \circ \mathcal{R}_{\mathcal{Y}} F(U)=\mathcal{R}_{\mathcal{Y}} F\left(V \times{ }_{Q} U\right) .
$$

Proof. (a) Let $l \in \mathcal{R}_{\mathcal{Y}} F(P)$, and $(T, S) \in \mathcal{Y}(P)$. The set $[T \backslash U / P]$ can be chosen to be equal to $\{1\}$, and for $u=1$, one has that $T^{u}=T$ and $S^{u}=S$. Thus

$$
\mathcal{R}_{\mathcal{Y}} F(U)(l)_{T, S}=(S \backslash T) \cdot l_{T, S}=l_{T, S},
$$

since $S \backslash T$ acts as the identity on $F(T / S)$.

(b) Let $l \in \mathcal{R}_{\mathcal{Y}} F(P)$ and $(T, S) \in \mathcal{Y}(R)$, and denote by $L$ the element $\left(\mathcal{R}_{\mathcal{Y}} F(V) \circ\right.$ $\left.\mathcal{R}_{\mathcal{Y}} F(U)(l)\right)_{T, S}$. Then

$$
\begin{aligned}
L & =\sum_{v \in[T \backslash V / Q]}(S \backslash T v) \cdot \mathcal{R}_{\mathcal{Y}} F(U)(l)_{T^{v}, S^{v}} \\
& =\sum_{\substack{v \in[T \backslash V / Q] \\
u \in\left[T^{v} \backslash U / P\right]}}(S \backslash T v) \times_{T^{v} / S^{v}}\left(S^{v} \backslash T^{v} u\right) \cdot l_{\left(T^{v}\right)^{u},\left(S^{v}\right)^{u}}
\end{aligned}
$$

But by Lemma 6.4. $\left(T^{v}\right)^{u}=T^{\left(v,{ }_{Q} u\right)}$ and $\left(S^{v}\right)^{u}=S^{\left(v{ }_{Q}{ }^{u)}\right.}$. Moreover when $v$ runs through $[T \backslash V / Q]$ and $u$ runs through $\left[T^{v} \backslash U / P\right]$, the element $\left(v,{ }_{Q} u\right)$ runs through a set of representatives of the set $R \backslash\left(V \times{ }_{Q} U\right) / P$, and by Lemma 6.5, there is an isomorphism of $\left(T / S, T^{\left(v,{ }_{Q} u\right)}\right)$-bisets

$$
(S \backslash T v) \times_{T^{v} / S^{v}}\left(S^{v} \backslash T^{v} u\right) \cong S \backslash\left(T v \times_{T^{v}} T^{v} u\right) .
$$

Moreover, there is an isomorphism of $\left(T, T^{\left(v,{ }_{Q} u\right)}\right)$-bisets

$$
T v \times_{T^{v}} T^{v} u \cong T\left(v,{ }_{Q} u\right),
$$


sending $\left(t v,_{T} v u\right)$ to $t t^{\prime}\left(v,{ }_{Q} u\right)$, for $t \in T$ and $x \in T^{v}$, where $t^{\prime} \in T$ is such that $t^{\prime} v=v x$. The inverse isomorphism maps $t\left(v,_{Q} u\right)$ to $\left(t v,_{T} v\right)$, for $t \in T$. In order to see that this is well-defined, replace $t$ by $t w$ where $w \in T$ stabilizes $\left(v,{ }_{Q} u\right)$. Then $\left(w v,{ }_{Q} u\right)=\left(v,{ }_{Q} u\right)$, so that there exists $y \in Q$ such that $w v=v y$ and $u=y^{-1} u$. We now see that $y \in T^{v}$ and it follows that

$$
\left(t w v,_{T^{v}} u\right)=\left(t v y,_{T^{v}} u\right)=\left(t v,_{T^{v}} y u\right)=\left(t v,_{T^{v}} u\right) .
$$

Thus finally

$$
\begin{aligned}
L & =\sum_{\left(v,{ }_{Q} u\right) \in\left[R \backslash\left(V \times{ }_{Q} U\right) / P\right]}\left(S \backslash T\left(v,_{Q} u\right)\right) \cdot l_{T^{\left(v, Q_{Q} u\right)}, S^{\left(v,{ }_{Q}\right.}{ }^{u)}} \\
& =\mathcal{R}_{\mathcal{Y}} F\left(V \times \times_{Q} U\right)(l)_{T, S},
\end{aligned}
$$

and the result follows.

6.14. Theorem. Let $F$ be a biset functor defined on $\mathcal{Y}$. The correspondences mapping a p-group $P$ to $\mathcal{R}_{\mathcal{Y}} F(P)$ and a $(Q, P)$-biset $U$ to $\mathcal{R}_{\mathcal{Y}} F(U)$ define a functor from $\mathcal{C}_{p}$ to $\mathcal{A} b$ (in other words a biset functor on p-groups).

Proof. This follows from Lemmas 6.11 and 6.13, together with the observation that the correspondence $U \mapsto \mathcal{R}_{\mathcal{Y}} F(U)$ is obviously additive with respect to the biset $U$, in the sense that if $U$ is a disjoint union $U=U_{1} \sqcup U_{2}$, then $\mathcal{R}_{\mathcal{Y}} F(U)=\mathcal{R}_{\mathcal{Y}} F\left(U_{1}\right)+\mathcal{R}_{\mathcal{Y}} F\left(U_{2}\right)$.

Recall that $\mathcal{F}$ denotes the category of biset functors (defined on all $p$-groups) and $\mathcal{F}_{\mathcal{Y}}$ the category of biset functors defined on $\mathcal{Y}$. Moreover $\mathcal{O}: \mathcal{F} \rightarrow \mathcal{F}_{\mathcal{Y}}$ denotes the forgetful functor.

6.15. Theorem. The correspondence $F \mapsto \mathcal{R}_{\mathcal{Y}} F$ is a functor $\mathcal{R}_{\mathcal{Y}}$ from $\mathcal{F}_{\mathcal{Y}}$ to $\mathcal{F}$, and this functor is right adjoint to the forgetful functor $\mathcal{O}_{\mathcal{Y}}$. Moreover the composition $\mathcal{O}_{\mathcal{Y}} \circ \mathcal{R}_{\mathcal{Y}}$ is isomorphic to the identity functor of $\mathcal{F}_{\mathcal{Y}}$.

Proof. We first show that the correspondence $F \mapsto \mathcal{R}_{\mathcal{Y}} F$ is functorial in $F$. A morphism $\varphi: F \rightarrow G$ in $\mathcal{F}_{\mathcal{Y}}$ is a collection of morphisms $\varphi_{Q}: F(Q) \rightarrow G(Q)$, for $Q$ in $\mathcal{Y}$, subject to the usual naturality conditions. (Note that throughout this proof, we write $\varphi_{Q}$ instead of $\varphi(Q)$ for simplicity of notation.) If $P$ is a $p$-group and $l \in \mathcal{R} \mathcal{Y} F(P)$, and if $(T, S) \in \mathcal{Y}(P)$, we set

$$
l_{T, S}^{\prime}=\varphi_{T / S}\left(l_{T, S}\right) .
$$

Since the maps $\varphi_{T / S}$ commute with deflation maps, restriction maps, and conjugation maps by the above naturality conditions, it is it clear that this defines an element $l^{\prime} \in \mathcal{R}_{\mathcal{Y}} G(P)$. Now the correspondence $l \mapsto l^{\prime}$ is a linear map

$$
\mathcal{R}_{\mathcal{Y}}(\varphi)_{P}: \mathcal{R}_{\mathcal{Y}} F(P) \rightarrow \mathcal{R}_{\mathcal{Y}} G(P)
$$

Suppose now that $P$ and $Q$ are $p$-groups, and that $U$ is a finite $(Q, P)$-biset. With the previous notation,

$$
\begin{aligned}
\mathcal{R}_{\mathcal{Y} G(U)\left(l^{\prime}\right)_{T, S}} & =\sum_{u \in[T \backslash U / P]}(S \backslash T u) \cdot \varphi_{T^{u} / S^{u}}\left(l_{T^{u}, S^{u}}\right) \\
& =\sum_{u \in[T \backslash U / P]} \varphi_{T / S}\left((S \backslash T u) \cdot l_{T^{u}, S^{u}}\right) \\
& =\varphi_{T / S}\left(\mathcal{R}_{\mathcal{Y}} F(U)(l)_{T, S}\right)
\end{aligned}
$$


This shows that $\mathcal{R}_{\mathcal{Y}} G(U) \circ \mathcal{R}_{\mathcal{Y}}(\varphi)_{P}=\mathcal{R}_{\mathcal{Y}}(\varphi)_{Q} \circ \mathcal{R}_{\mathcal{Y}} F(U)$. In other words, the maps $\mathcal{R}_{\mathcal{Y}}(\varphi)_{P}$ define a natural transformation of functors $\mathcal{R}_{\mathcal{Y}}(\varphi): \mathcal{R}_{\mathcal{Y}} F \rightarrow \mathcal{R}_{\mathcal{Y}} G$. It is now clear that the correspondence $F \mapsto \mathcal{R}_{\mathcal{Y}} F$ is a functor $\mathcal{F}_{\mathcal{Y}} \rightarrow \mathcal{F}$.

It is convenient to prove the last assertion of the theorem now. We have to show that if $F \in \mathcal{F}_{\mathcal{Y}}$ and if $Q$ is in the class $\mathcal{Y}$, then there is an isomorphism

$$
\varepsilon_{Q}: F(Q) \rightarrow \mathcal{O}_{\mathcal{Y}} \mathcal{R}_{\mathcal{Y}} F(Q)
$$

which is natural with respect to $F$ and $Q$. This isomorphism is defined as follows. If $f \in F(Q)$ and $(T, S) \in \mathcal{Y}(Q)$, then $\varepsilon_{Q}(f)_{T, S}=\operatorname{Defres}_{T / S}^{Q} f$. It is routine to check that this definition gives the required isomorphism of functors id $\rightarrow \mathcal{O}_{\mathcal{Y}} \circ \mathcal{R}_{\mathcal{Y}}$.

We turn now to the adjointness property. Suppose that $F \in \mathcal{F}, G \in \mathcal{F}_{\mathcal{Y}}$, and $\varphi: \mathcal{O}_{\mathcal{Y}} F \rightarrow G$ is a morphism in $\mathcal{F}_{\mathcal{Y}}$. Thus $\varphi$ is a collection of morphisms $\varphi_{Q}: F(Q) \rightarrow$ $G(Q)$, for $Q \in \mathcal{Y}$, satisfying some commutation conditions. Now if $P$ is any $p$-group, $f \in F(P)$, and $(T, S) \in \mathcal{Y}(P)$, set

$$
l_{T, S}=\varphi_{T / S}\left(\operatorname{Defres}_{T / S}^{P} f\right) .
$$

The above commutation conditions show easily that these elements $l_{T, S}$ define an element $l$ of $\mathcal{R}_{\mathcal{Y}} G(P)$. Now the correspondence $f \mapsto l$ is a linear map from $F(P)$ to $\mathcal{R}_{\mathcal{Y}} G(P)$, denoted by $\varphi_{P}^{+}$, such that

$$
\varphi_{P}^{+}(f)_{T, S}=\varphi_{T / S}\left(\operatorname{Defres}_{T / S}^{P} f\right)
$$

for any $p$-group $P$, any $f \in F(P)$, and any $(T, S) \in \mathcal{Y}(P)$. We now show that the maps $\varphi_{P}^{+}$define a morphism $\varphi^{+}: F \rightarrow \mathcal{R}_{\mathcal{Y}} G$ in $\mathcal{F}$.

If $Q$ is a $p$-group and $U$ is a finite $(Q, P)$-biset, then for each $f \in F(P)$ and each $(T, S) \in \mathcal{Y}(Q)$, one has that

$$
\begin{aligned}
\left(\mathcal{R}_{\mathcal{Y}} G(U) \circ \varphi_{P}^{+}(f)\right)_{T, S} & =\sum_{u \in[T \backslash U / P]}(S \backslash T u) \cdot \varphi_{T^{u} / S^{u}}\left(\operatorname{Defres}_{T^{u} / S^{u}}^{P} f\right) \\
& =\sum_{u \in[T \backslash U / P]} \varphi_{T / S}\left((S \backslash T u) \cdot \operatorname{Defres}_{T^{u} / S^{u}}^{P} f\right) \\
& =\varphi_{T / S}\left(\sum_{u \in[T \backslash U / P]}(S \backslash T u) \times_{T^{u} / S^{u}}\left(S^{u} \backslash P\right) \cdot f\right) .
\end{aligned}
$$

By Lemma 6.5 there is an isomorphism of $(T / S, P)$-bisets

$$
(S \backslash T u) \times_{T^{u} / S^{u}}\left(S^{u} \backslash P\right) \cong S \backslash\left(T u \times_{T^{u}} P\right) .
$$


Moreover $T u \times_{T^{u}} P \cong T u P$ as $(T, P)$-bisets. Thus

$$
\begin{aligned}
\left(\mathcal{R}_{\mathcal{Y}} G(U) \circ \varphi_{P}^{+}(f)\right)_{T, S} & =\varphi_{T / S}\left(\sum_{u \in[T \backslash U / P]} S \backslash T u P \cdot f\right) \\
& =\varphi_{T / S}\left(\left(\bigsqcup_{u \in[T \backslash U / P]} S \backslash T u P\right) \cdot f\right) \\
& =\varphi_{T / S}((S \backslash U) \cdot f) \\
& =\varphi_{T / S}\left(\left(S \backslash Q \times_{Q} U\right) \cdot f\right) \\
& =\varphi_{T / S}\left(\operatorname{Defres}_{T / S}^{Q}(U \cdot f)\right) \\
& =\left(\varphi_{Q}^{+}(U \cdot f)\right)_{T, S} \\
& =\left(\varphi_{Q}^{+} F(U)(f)\right)_{T, S} .
\end{aligned}
$$

This shows that $\mathcal{R}_{\mathcal{y}} G(U) \circ \varphi_{P}^{+}=\varphi_{Q}^{+} \circ F(U)$, so the maps $\varphi_{P}^{+}$define a morphism $\varphi^{+}: F \rightarrow \mathcal{R}_{\mathcal{Y}} G$ in $\mathcal{F}$.

Conversely, suppose that $\psi: F \rightarrow \mathcal{R}_{\mathcal{Y}} G$ is a morphism in $\mathcal{F}$. Then $\mathcal{O}_{\mathcal{Y}}(\psi)$ is a morphism from $\mathcal{O}_{\mathcal{Y}} F$ to $\mathcal{O}_{\mathcal{Y}} \mathcal{R}_{\mathcal{Y}} G$, and the latter is isomorphic to $G$. It is easy to check that the corresponding morphism $\psi^{-}: \mathcal{O}_{\mathcal{Y}} F \rightarrow G$ maps the element $f \in F(Q)$, for $Q \in \mathcal{Y}$, to $\psi_{Q}(f)_{Q, 1}$, where we have identified $Q / 1$ with $Q$. In other words

$$
\psi_{Q}^{-}(f)=\psi_{Q}(f)_{Q, 1}
$$

for any $Q \in \mathcal{Y}$ and any $f \in F(Q)$.

We have now defined morphisms

$$
\begin{array}{ll}
\operatorname{Hom}_{\mathcal{F}_{\mathcal{Y}}}\left(\mathcal{O}_{\mathcal{Y}} F, G\right) \longrightarrow \operatorname{Hom}_{\mathcal{F}}\left(F, \mathcal{R}_{\mathcal{Y}} G\right), & \\
\operatorname{Hom}_{\mathcal{F}}\left(F, \mathcal{R}_{\mathcal{Y}} G\right) \longrightarrow \operatorname{Hom}_{\mathcal{F}_{\mathcal{Y}}}\left(\mathcal{O}_{\mathcal{Y}} F, G\right), & \psi \mapsto \varphi^{+} \\
\hline
\end{array}
$$

and we have to show that they are mutual inverse. If $\varphi: \mathcal{O}_{\mathcal{Y}} F \rightarrow G$ is a morphism, then for any $Q \in \mathcal{Y}$ and $f \in F(Q)$, we have

$$
\left(\varphi^{+}\right)_{Q}^{-}(f)=\varphi_{Q}^{+}(f)_{Q, 1}=\varphi_{Q / 1}\left(\operatorname{Defres}_{Q / 1}^{Q} f\right)=\varphi_{Q}(f) .
$$

Hence $\left(\varphi^{+}\right)^{-}=\varphi$.

Conversely, if $\psi: F \rightarrow \mathcal{R}_{\mathcal{Y}} G$ is a morphism, $P$ is a p-group, $f \in F(P)$, and $(T, S) \in \mathcal{Y}(P)$, then

$$
\begin{aligned}
\left(\psi^{-}\right)_{P}^{+}(f)_{T, S} & =\psi_{T / S}^{-}\left(\operatorname{Defres}_{T / S}^{P} f\right) \\
& =\left(\psi_{T / S} \operatorname{Defres}_{T / S}^{P} f\right)_{T / S, 1} \\
& =\left(\operatorname{Defres}_{T / S}^{P} \psi_{P}(f)\right)_{T / S, S / S} \\
& =\left(\psi_{P}(f)\right)_{T / S, S / S}=\psi_{P}(f)_{T, S},
\end{aligned}
$$

using Example 6.10 and the identification between $(T / S) /(S / S)$ and $T / S$. Therefore $\left(\psi^{-}\right)^{+}=\psi$.

Thus we have proved that the correspondences $\varphi \mapsto \varphi^{+}$and $\psi \mapsto \psi^{-}$provide mutual inverse isomorphisms

$$
\operatorname{Hom}_{\mathcal{F}_{\mathcal{Y}}}\left(\mathcal{O}_{\mathcal{Y}} F, G\right) \leftrightarrows \operatorname{Hom}_{\mathcal{F}}\left(F, \mathcal{R}_{\mathcal{Y}} G\right)
$$


We leave to the reader the easy verification that these isomorphisms are natural in $F$ and $G$.

6.17. Corollary of proof. Let $F \in \mathcal{F}$ and let $P$ be a p-group. Let $\eta_{F}^{\mathcal{Y}}: F \longrightarrow$ $\mathcal{R}_{y} \mathcal{O}_{\mathcal{Y}} F$ be the unit morphism associated with the adjunction of Theorem 6.15. Then the evaluation $\eta_{F}^{\mathcal{Y}}(P)$ coincides with the map

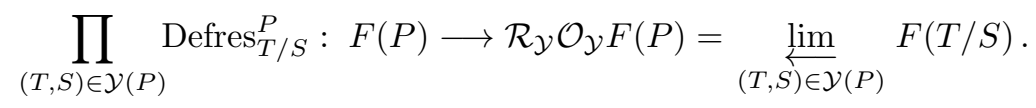

Proof. Via the adjunction, the unit morphism $\eta_{F}^{\mathcal{Y}}$ corresponds to the identity id : $\mathcal{O}_{\mathcal{Y}} F \rightarrow \mathcal{O}_{\mathcal{Y}} F$, that is, $\eta_{F}^{\mathcal{Y}}=\mathrm{id}^{+}$. But for any $f \in F(P), \mathrm{id}_{P}^{+}(f)$ is defined by the expression (6.16) in the proof above, that is, the family $\operatorname{Defres}_{T / S}^{P} f$, where $(T, S)$ runs through $\mathcal{Y}(P)$.

\section{References}

[Bo1] S. Bouc. Foncteurs d'ensembles munis d'une double action, J. Algebra 183 (1996), 664-736.

[Bo2] S. Bouc. Tensor induction of relative syzygies, J. reine angew. Math. 523 (2000), 113-171.

[Bo3] S. Bouc. A remark on the Dade group and the Burnside group, J. Algebra 279 (2004), 180-190.

[Bo4] S. Bouc. The Dade group of a p-group, Invent. Math. 164 (2006), 189-231.

[Bo5] S. Bouc. Gluing endo-permutation modules, in preparation.

[BoTh1] S. Bouc, J. Thévenaz. The group of endo-permutation modules, Invent. Math. 139 (2000), 275-349.

[BoTh2] S. Bouc, J. Thévenaz. Gluing torsion endo-permutation modules. Preprint, 2006.

[CaTh1] J. Carlson, J. Thévenaz. The classification of torsion endo-trivial modules, Ann. of Math. 162 (2005), 823-883.

[CaTh2] J. Carlson, J. Thévenaz. The classification of endo-trivial modules, Invent. Math. 158 (2004), 389-411.

[GZ] P. Gabriel, M. Zisman. Calculus of fractions and homotopy theory, Ergebnisse der Mathematik 35, Springer Verlag, Berlin-Heidelberg, 1967.

[Th] J. Thévenaz. Endo-permutation modules, a guided tour, in Group Representation Theory, edited by M. Geck, D. Testerman, J. Thévenaz, EPFL Press, Lausanne, 2007. 
Serge Bouc, CNRS-LAMFA, Université de Picardie - Jules Verne, 33, rue St Leu, F-80039 Amiens Cedex 1, France.

serge.bouc@u-picardie.fr

Jacques Thévenaz, Institut de Géométrie, Algèbre et Topologie, EPFL, Bâtiment BCH, CH-1015 Lausanne, Switzerland.

Jacques. Thevenaz@epfl.ch 